\title{
The Real $K$-Theory of Compact Lie Groups
}

\author{
Chi-Kwong FOK
}

Department of Mathematics, Cornell University, Ithaca, NY 14853, USA

E-mail: ckfok@math.cornell.edu

URL: http://www. math. cornell.edu/ ckfok/

Received August 22, 2013, in final form March 06, 2014; Published online March 11, 2014

http://dx.doi.org/10.3842/SIGMA.2014.022

\begin{abstract}
Let $G$ be a compact, connected, and simply-connected Lie group, equipped with a Lie group involution $\sigma_{G}$ and viewed as a $G$-space with the conjugation action. In this paper, we present a description of the ring structure of the (equivariant) $K R$-theory of $\left(G, \sigma_{G}\right)$ by drawing on previous results on the module structure of the $K R$-theory and the ring structure of the equivariant $K$-theory.
\end{abstract}

Key words: KR-theory; compact Lie groups; Real representations; Real equivariant formality

2010 Mathematics Subject Classification: 19L47; 57T10

\section{Introduction}

The complex $K$-theory of compact connected Lie groups with torsion-free fundamental groups was worked out by Hodgkin in the 60s (cf. [13] and Theorem 2.4). It asserts that the $K$-theory ring is the $\mathbb{Z}_{2}$-graded exterior algebra over $\mathbb{Z}$ on the module of primitive elements, which are of degree -1 and associated with the representations of the Lie group. For the elegant proof of Hodgkin's result in the special case where $G$ is simply-connected, see [1].

In [2], Atiyah introduced $K R$-theory, which is basically a version of topological $K$-theory for the category of Real spaces, i.e. topological spaces equipped with an involution. $K R$-theory can be regarded as a hybrid of $K O$-theory, complex $K$-theory and $K S C$-theory (cf. [2]). One can also consider equivariant $K R$-theory, where a certain compatibility condition between the group action and the involution is assumed. For definitions and some basic properties, see Definitions 2.26 and $2.27[2,5]$.

Since Hodgkin's work, there have appeared two kinds of generalizations of $K$-theory of compact Lie groups. The first such is $K R$-theory of compact Lie groups, which was first studied by Seymour (cf. [15]). He obtained the $K R^{*}(\mathrm{pt})$-module structure of $K R^{*}(G)$, where $G$ is a compact, connected and simply-connected Lie group equipped with a Lie group involution, using his structure theorem of $K R$-theory of a certain type of spaces (cf. Theorems 2.32, 2.37 and 2.40). He was unable to obtain a complete description of the ring structure, however, and could only make some conjectures about it. In [7], Bousfield determined functorially the united 2-adic $K$ cohomology algebra of any compact, connected and simply-connected Lie group, which includes the 2-adic KO-cohomology algebra, and hence extended Seymour's results in the 2-adic case, if the Lie group involution is taken into account appropriately.

The second one is the equivariant $K$-theory of compact Lie groups. In [9], Brylinski and Zhang showed that, for a compact connected Lie group $G$ with torsion free fundamental group, the equivariant $K$-theory, $K_{G}^{*}(G)$, where $G$ acts on itself by conjugation, is isomorphic to the ring of Grothendieck differentials of the complex representation ring $R(G)$ (for definition, see [9]). It is noteworthy that $G$ satisfies the property of being weakly equivariantly formal $\grave{a}$ la Harada and Landweber (cf. Definition 4.1 of [11] and Remark 2.8). 
In this paper, based on the previous works of Seymour's and Brylinski-Zhang's and putting both the Real and equivariant structures together, we obtain a description of the ring structure of the equivariant $K R$-theory of any compact, connected and simply-connected Lie group, which is recorded in Theorem 4.33. We express the ring structure using relations of generators associated to Real representations of $G$ of real, complex and quaternionic type (with respect to the Lie group involution). Our main contribution is twofold. First, we observe that the conditions of Seymour's structure theorem are an appropriate candidate for defining the notion of 'Real formality' in analogy to weakly equivariant formality. These notions together prompt us to introduce the definition of 'Real equivariant formality', which leads to a structure theorem for equivariant $K R$-theory (Theorem 4.5). Any compact, connected and simply-connected Real Lie group falls under the category of Real equivariantly formal spaces and Theorem 4.5 enables us to obtain a preliminary description of the equivariant $K R$-theory as an algebra over the coefficient ring. Second, inspired by Seymour's conjecture, we obtain the squares of the real and quaternionic type generators, which in addition to other known relations complete the description of the ring structure. These squares are non-zero 2-torsions in general. Hence the equivariant $K R$-theory in general is not a ring of Grothendieck differentials, as in the case for equivariant $K$-theory. Despite this, we remark that, in certain cases, if we invert 2 in the equivariant $K R$-theory ring, then the result is an exterior algebra over the localized coefficient ring of equivariant $K R$-theory.

The organization of this paper is as follows. In Section 2, we review the (equivariant) $K$-theory of compact connected Lie groups with torsion-free fundamental groups, Real representation rings $R R(G), K R$-theory, and the main results in [15]. In Section 3 we give a description of the coefficient ring $K R_{G}^{*}(\mathrm{pt})$. Section 4 is devoted to proving Theorems 4.5 and 4.33 , the main results of this paper, which give a full description of the ring structure of $K R_{G}^{*}(G)$. In Section 5 we apply the main results to obtain the ring structure of the ordinary $K R$-theory of compact Lie groups, thereby confirming Seymour's conjecture on the squares of the real type generators and disproving that on the squares of the quaternionic type generators. We also work out several examples, one of which shows how equivariant $K R$-theory tells apart (while $K$-theory cannot) the ring structures of two equivariant $K R$-theory rings of $G$, where in one case $G$ acts on itself trivially, while in another $G$ acts by conjugation.

Throughout this paper, we follow the convention in [2] and [5] in reference to $K R$-theory. In particular, we use the word 'Real' (with a capital R) in all contexts involving involutions, so as to avoid confusion with the word 'real' with the usual meaning. For example, 'Real $K$-theory' is used interchangeably with $K R$-theory, whereas 'real $K$-theory' means $K O$-theory.

\section{Preliminaries}

Throughout this section, $G$ denotes any compact Lie groups, and $X, Y, \ldots$ any finite $C W$ complexes unless otherwise specified.

\section{1 (Equivariant) $K$-theory of compact connected Lie groups}

Recall that the functor $K^{-1}$ is represented by $U(\infty):=\lim _{n \rightarrow \infty} U(n)$, i.e. for any $X, K^{-1}(X)$ is the abelian group of homotopy classes of maps $[X, U(\infty)]$. Such a description of $K^{-1}$ leads to the following

Definition 2.1. Let $\delta: R(G) \rightarrow K^{-1}(G)$ be the group homomorphism which sends any complex $G$-representation $\rho$ to the homotopy class of $i \circ \rho$, where $\rho$ is regarded as a continuous map from $G$ to $U(n)$ and $i: U(n) \hookrightarrow U(\infty)$ is the standard inclusion.

In fact, any element in $K^{-q}(X)$ can be represented by a complex of vector bundles on $X \times \mathbb{R}^{q}$, which is exact outside $X \times\{0\}$ (cf. [3]). This gives another interpretation of $\delta(\rho)$, as shown in the following proposition, which we find useful for our exposition. 
Proposition 2.2. If $V$ is the underlying complex vector space of $\rho$, then $\delta(\rho)$ is represented by

$$
\begin{aligned}
& 0 \rightarrow G \times \mathbb{R} \times V \rightarrow G \times \mathbb{R} \times V \rightarrow 0, \\
& (g, t, v) \mapsto(g, t,-t \rho(g) v) \quad \text { if } t \geq 0, \\
& (g, t, v) \mapsto(g, t, t v) \quad \text { if } t \leq 0 .
\end{aligned}
$$

\section{Proposition 2.3 ([13]).}

1. $\delta$ is a derivation of $R(G)$ taking values in $K^{-1}(G)$ regarded as an $R(G)$-module whose module structure is given by the augmentation map. In other words, $\delta$ is a group homomorphism from $R(G)$ to $K^{-1}(G)$ satisfying

$$
\delta\left(\rho_{1} \otimes \rho_{2}\right)=\operatorname{dim}\left(\rho_{1}\right) \delta\left(\rho_{2}\right)+\operatorname{dim}\left(\rho_{2}\right) \delta\left(\rho_{1}\right) .
$$

2. If $I(G)$ is the augmentation ideal of $R(G)$, then $\delta\left(I(G)^{2}\right)=0$.

The main results of [13] are stated in the following

Theorem 2.4. Let $G$ be a compact connected Lie group with torsion-free fundamental group. Then

1. $K^{*}(G)$ is torsion-free.

2. Let $J(G):=I(G) / I(G)^{2}$. Then the map $\widetilde{\delta}: J(G) \rightarrow K^{-1}(G)$ induced by $\delta$ is well-defined, and $K^{*}(G)=\bigwedge(\operatorname{Im}(\widetilde{\delta}))$.

3. In particular, if $G$ is compact, connected and simply-connected of rank $l$, then $K^{*}(G)=$ $\bigwedge_{\mathbb{Z}}\left(\delta\left(\rho_{1}\right), \ldots, \delta\left(\rho_{l}\right)\right)$, where $\rho_{1}, \ldots, \rho_{l}$ are the fundamental representations.

Viewing $G$ as a $G$-space via the adjoint action, one may consider the equivariant $K$-theory $K_{G}^{*}(G)$. Let $\Omega_{R(G) / \mathbb{Z}}^{*}$ be the ring of Grothendieck differentials of $R(G)$ over $\mathbb{Z}$, i.e. the exterior algebra over $R(G)$ of the module of Kähler differentials of $R(G)$ over $\mathbb{Z}$ (cf. [9]).

Definition 2.5. Let $\varphi: \Omega_{R(G) / \mathbb{Z}}^{*} \rightarrow K_{G}^{*}(G)$ be the $R(G)$-algebra homomorphism defined by the following

1) $\varphi\left(\rho_{V}\right):=[G \times V] \in K_{G}^{*}(G)$, where $G$ acts on $G \times V$ by $g_{0} \cdot\left(g_{1}, v\right)=\left(g_{0} g_{1} g_{0}^{-1}, \rho_{V}\left(g_{0}\right) v\right)$,

2) $\varphi\left(d \rho_{V}\right) \in K_{G}^{-1}(G)$ is the complex of vector bundles in Definition 2.2 where $G$ acts on $G \times \mathbb{R} \times V$ by $g_{0} \cdot\left(g_{1}, t, v\right)=\left(g_{0} g_{1} g_{0}^{-1}, t, \rho_{V}\left(g_{0}\right) v\right)$,

We also define $\delta_{G}: R(G) \rightarrow K_{G}^{-1}(G)$ by $\delta_{G}\left(\rho_{V}\right):=\varphi\left(d \rho_{V}\right)$.

Remark 2.6. The definition of $\delta_{G}\left(\rho_{V}\right)$ given in [9], where the middle map of the complex of vector bundles is defined to be $(g, t, v) \mapsto\left(g, t, t \rho_{V}(g) v\right)$ for all $t \in \mathbb{R}$, is incorrect, as $\delta_{G}\left(\rho_{V}\right)$ so defined is actually 0 in $K_{G}^{-1}(G)$. The definition given in Definition 2.5 is the correction made by Brylinski and relayed to us by one of the referees.

\section{Theorem $2.7([9])$.}

1. $\delta_{G}$ is a derivation of $R(G)$ taking values in the $R(G)$-module $K_{G}^{-1}(G)$, i.e. $\delta_{G}$ satisfies

$$
\delta_{G}\left(\rho_{1} \otimes \rho_{2}\right)=\rho_{1} \cdot \delta_{G}\left(\rho_{2}\right)+\rho_{2} \cdot \delta_{G}\left(\rho_{1}\right) .
$$

2. Let $G$ be a compact connected Lie group with torsion-free fundamental group. Then $\varphi$ is an $R(G)$-algebra isomorphism. 


\section{Remark 2.8.}

1. Although the definition of $\delta_{G}\left(\rho_{V}\right)$ given by Brylinski-Zhang in [9] is incorrect, their proof of Theorem 2.7 can be easily corrected by using the correct definition as in Definition 2.5, which does not affect the validity of the rest of their arguments, and Theorem 2.7 still stands.

2. In [11], a $G$-space $X$ is defined to be weakly equivariantly formal if the map $K_{G}^{*}(X) \otimes_{R(G)}$ $\mathbb{Z} \rightarrow K^{*}(X)$ induced by the forgetful map is a ring isomorphism, where $\mathbb{Z}$ is viewed as an $R(G)$-module through the augmentation homomorphism. Theorem 2.4 and 2.7 imply that $G$ is weakly equivariantly formal if it is connected with torsion-free fundamental group. We will make use of this property in computing the equivariant $K R$-theory of $G$.

3. Let $f: K_{G}^{*}(G) \rightarrow K^{*}(G)$ be the forgetful map. Note that $f(\varphi(\rho))=\operatorname{dim}(\rho)$ and $f(\varphi(d \rho))=\delta(\rho)$. Applying $f$ to equation (2.2) in Theorem 2.7, we get equation (2.1) in Proposition 2.3.

4. $I(G)$, being a prime ideal in $R(G)$, can be thought of as an element in $\operatorname{Spec} R(G)$, and $K^{*}(G) \cong \bigwedge_{\mathbb{Z}} T_{I(G)}^{*} \operatorname{Spec} R(G), K_{G}^{*}(G) \cong \bigwedge_{R(G)} T_{I(G)}^{*} \operatorname{Spec} R(G)$.

\subsection{Real representation rings}

This section is an elaboration of the part on Real representation rings in [5] and [15]. Since the results in this subsection can be readily generalized from those results concerning the special case where $\sigma_{G}$ is trivial, we omit most of the proofs and refer the reader to any standard text on representation theory of Lie groups, e.g. [6] and [8].

Definition 2.9. A Real Lie group is a pair $\left(G, \sigma_{G}\right)$ where $G$ is a Lie group and $\sigma_{G}$ a Lie group involution on it. A Real representation $V$ of a Real Lie group $\left(G, \sigma_{G}\right)$ is a finite-dimensional complex representation of $G$ equipped with an anti-linear involution $\sigma_{V}$ such that $\sigma_{V}(g v)=$ $\sigma_{G}(g) \sigma_{V}(v)$. Let $\operatorname{Rep}_{\mathbb{R}}\left(G, \sigma_{G}\right)$ be the category of Real representations of $\left(G, \sigma_{G}\right)$. A morphism between $V$ and $W \in \mathcal{R} p_{\mathbb{R}}\left(G, \sigma_{G}\right)$ is a linear transformation from $V$ to $W$ which commute with $G$ and respect $\sigma_{V}$ and $\sigma_{W}$. We denote $\operatorname{Mor}(V, W)$ by $\operatorname{Hom}_{G}(V, W)^{\left(\sigma_{V}, \sigma_{W}\right)}$. An irreducible Real representation is an irreducible object in $\mathcal{R e p}_{\mathbb{R}}\left(G, \sigma_{G}\right)$. The Real representation ring of $\left(G, \sigma_{G}\right)$, denoted by $R R\left(G, \sigma_{G}\right)$, is the Grothendieck group of $\mathcal{R} e p_{\mathbb{R}}\left(G, \sigma_{G}\right)$, with multiplication being tensor product over $\mathbb{C}$. Sometimes we will omit the notation $\sigma_{G}$ if there is no danger of confusion about the Lie group involution.

Remark 2.10. Let $V$ be an irreducible Real representation of $G$. Then $\operatorname{Hom}_{G}(V, V)^{\sigma_{V}}$ must be a real associative division algebra which, according to Frobenius' theorem, is isomorphic to either $\mathbb{R}, \mathbb{C}$ or $\mathbb{H}$. Following the convention of [5], we call $\operatorname{Hom}_{G}(V, V)^{\sigma_{V}}$ the commuting field of $V$.

Definition 2.11. If $V$ is an irreducible Real representation of $G$, then we say $V$ is of real, complex or quaternionic type according as the commuting field is isomorphic to $\mathbb{R}, \mathbb{C}$ or $\mathbb{H}$. Let $R R(G, \mathbb{F})$ be the abelian group generated by the isomorphism classes of irreducible Real representations with $\mathbb{F}$ as the commuting field.

Remark 2.12. $R R(G) \cong R R(G, \mathbb{R}) \oplus R R(G, \mathbb{C}) \oplus R R(G, \mathbb{H})$ as abelian groups.

Definition 2.13. Let $V$ be a $G$-representation. We use $\sigma_{G}^{*} V$ to denote the $G$-representation with the same underlying vector space where the $G$-action is twisted by $\sigma_{G}$, i.e. $\rho_{\sigma_{G}^{*} V}(g) v=$ $\rho_{V}\left(\sigma_{G}(g)\right) v$. We will use $\overline{\sigma_{G}^{*}}$ to denote the map on $R(G)$ defined by $[V] \mapsto\left[\sigma_{G}^{*} \bar{V}\right]$.

Proposition 2.14. If $V$ is a complex $G$-representation, and there exists $f \in \operatorname{Hom}_{G}\left(V, \sigma_{G}^{*} \bar{V}\right)$ such that $f^{2}=\operatorname{Id}_{V}$, then $V$ is a Real representation of $G$ with $f$ as the anti-linear involution $\sigma_{V}$. 
Proof. If $f \in \operatorname{Hom}_{G}\left(V, \sigma_{G}^{*} \bar{V}\right)$, then it is anti-linear on $V$ and $f(g v)=\sigma_{G}(g) f(v)$ for $g \in G$ and $v \in V$. The assumption that $f^{2}=\operatorname{Id}_{V}$ just says that $f$ is an involution. So $V$ together with $\sigma_{V}=f$ is a Real representation of $G$.

Proposition 2.15. Let $V$ be an irreducible complex representation of $G$ and suppose that $V \cong$ $\sigma_{G}^{*} \bar{V}$. Let $f \in \operatorname{Hom}_{G}\left(V, \sigma_{G}^{*} \bar{V}\right)$. Then

1. $f^{2}=k \operatorname{Id}_{V}$ for some $k \in \mathbb{R}$.

2. There exists $g \in \operatorname{Hom}_{G}\left(V, \sigma_{G}^{*} \bar{V}\right)$ such that $g^{2}=\operatorname{Id}_{V}$ or $g^{2}=-\operatorname{Id}_{V}$.

Proof. Note that $f^{2} \in \operatorname{Hom}_{G}(V, V)$. By Schur's lemma, $f^{2}=k \operatorname{Id}_{V}$ for some $k \in \mathbb{C}$. On the other hand,

$$
f(k v)=f(f(f(v)))=k f(v) .
$$

But $f$ is an anti-linear map on $V$. It follows that $k=\bar{k}$ and hence $k \in \mathbb{R}$. For part (2), we may first simply pick an isomorphism $f \in \operatorname{Hom}_{G}\left(V, \sigma_{G}^{*} \bar{V}\right)$. Then $f^{2}=k \operatorname{Id}_{V}$ for some $k \in \mathbb{R}^{\times}$. Schur's lemma implies that any $g \in \operatorname{Hom}_{G}\left(V, \sigma_{G}^{*} \bar{V}\right)$ must be of the form $g=c f$ for some $c \in \mathbb{C}$. Then $g^{2}=c f \circ c f=c \bar{c} f^{2}=|c|^{2} k \operatorname{Id}_{V}$. Consequently, if $k$ is positive, we choose $c=\frac{1}{\sqrt{k}}$ so that $g^{2}=\operatorname{Id}_{V} ;$ if $k$ is negative, we choose $c=\frac{1}{\sqrt{-k}}$ so that $g^{2}=-\operatorname{Id}_{V}$.

Proposition 2.16. Let $V$ be an irreducible Real representation of $G$.

1. The commuting field of $V$ is isomorphic to $\mathbb{R}$ iff $V$ is an irreducible complex representation and there exists $f \in \operatorname{Hom}_{G}\left(V, \sigma_{G}^{*} \bar{V}\right)$ such that $f^{2}=\operatorname{Id}_{V}$.

2. The commuting field of $V$ is isomorphic to $\mathbb{C}$ iff $V \cong W \oplus \sigma_{G}^{*} \bar{W}$ as complex $G$-representations, where $W$ is an irreducible complex $G$-representation and $W ¥ \sigma_{G}^{*} \bar{W}$, and $\sigma_{V}\left(w_{1}, w_{2}\right)$ $=\left(w_{2}, w_{1}\right)$.

3. The commuting field of $V$ is isomorphic to $\mathbb{H}$ iff $V \cong W \oplus \sigma_{G}^{*} \bar{W}$ as complex $G$-representations, where $W$ is an irreducible complex $G$-representation and there exists $f \in$ $\operatorname{Hom}_{G}\left(V, \sigma_{G}^{*} \bar{V}\right)$ such that $f^{2}=-\operatorname{Id}_{V}$, and $\sigma_{V}\left(w_{1}, w_{2}\right)=\left(w_{2}, w_{1}\right)$.

Proof. One can easily establish the above proposition by modifying the proof of Proposition 3 in Appendix 2 of [6], which is a special case of the above proposition where $\sigma_{G}$ is trivial.

\section{Proposition 2.17.}

1. The map $i: R R(G) \rightarrow R(G)$ which forgets the Real structure is injective.

2. Any complex $G$-representation $V$ which is a Real representation can only possess a unique Real structure up to isomorphisms of Real G-representations.

Proof. Let $\rho: R(G) \rightarrow R R(G)$ be the map

$$
[V] \mapsto\left[\left(V \oplus \iota_{G}^{*} \bar{V}, \sigma_{V \oplus \iota_{G}^{*} \bar{V}}\right)\right],
$$

where $\sigma_{V \oplus \iota_{G}^{*} \bar{V}}(u, w)=(w, u)$. Let $\left[\left(V, \sigma_{V}\right)\right] \in R R(G)$. Then

$$
\rho \circ i\left(\left[\left(V, \sigma_{V}\right)\right]\right)=\left[\left(V \oplus \iota_{G}^{*} \bar{V}, \sigma_{V \oplus \iota_{G}^{*} \bar{V}}\right)\right] .
$$

We claim that $\left[\left(V \oplus V, \sigma_{V} \oplus \sigma_{V}\right)\right]=\left[\left(V \oplus \iota_{G}^{*} \bar{V}, \sigma_{V \oplus \iota_{G}^{*} \bar{V}}\right)\right]$, which is easily seen to be true because of the Real $G$-representation isomorphism

$$
\begin{aligned}
f: & V \oplus \iota_{G}^{*} \bar{V} \rightarrow V \oplus V, \\
& (u, w) \mapsto\left(u+\sigma_{V}(w), i\left(u-\sigma_{V}(w)\right)\right) .
\end{aligned}
$$


It follows that $\rho \circ i$ amounts to multiplication by 2 on $R R(G)$, and is therefore injective because $R R(G)$ is a free abelian group generated by irreducible Real representations. Hence $i$ is injective. $(2)$ is simply a restatement of (1).

Proposition 2.17 makes it legitimate to regard $R R(G)$ as a subring of $R(G)$. From now on we view $[V] \in R(G)$ as an element in $R R(G)$ if $V$ possesses a compatible Real structure.

Proposition 2.18. Let $G$ be a compact Real Lie group. Let $V$ be an irreducible complex representation of $G$. Then

1. $[V] \in R R(G, \mathbb{R})$ iff there exists a $G$-invariant symmetric nondegenerate bilinear form $B$ : $V \times \sigma_{G}^{*} V \rightarrow \mathbb{C}$.

2. $[V \oplus V] \in R R(G, \mathbb{H})$ iff there exists a $G$-invariant skew-symmetric nondegenerate bilinear form $B: V \times \sigma_{G}^{*} V \rightarrow \mathbb{C}$.

3. $\left[V \oplus \sigma_{G}^{*} \bar{V}\right] \in R R(G, \mathbb{C})$ iff there does not exist any $G$-invariant nondegenerate bilinear form on $V \times \sigma_{G}^{*} V$.

Proof. By Proposition 2.16, $V$ is a Real representation of real type iff there exists $f \in$ $\operatorname{Hom}\left(V, \sigma_{G}^{*} \bar{V}\right)^{G}$ such that $f^{2}=\operatorname{Id}_{V}$. One can define a bilinear form $B: V \times \sigma_{G}^{*} V \rightarrow \mathbb{C}$ by

$$
B\left(v_{1}, v_{2}\right)=\left\langle v_{1}, f\left(v_{2}\right)\right\rangle
$$

where $\langle$,$\rangle is a G$-invariant Hermitian inner product on $V$. It can be easily seen that $B$ is $G$-invariant, symmetric and non-degenerate. Conversely, given a $G$-invariant symmetric nondegenerate bilinear form on $V \times \sigma_{G}^{*} V$ and using equation (2.3), we can define $f \in \operatorname{Hom}\left(V, \sigma_{G}^{*} \bar{V}\right)^{G}$, which squares to identity. Part (2) and (3) follow similarly.

Proposition 2.18 leads to the following

Definition 2.19. Let $G$ be a compact Real Lie group. Let $V$ be an irreducible complex representation of $G$. Define, with respect to $\sigma_{G}$,

1. $V$ to be of real type if there exists a $G$-invariant symmetric nondegenerate bilinear form $B: V \times \sigma_{G}^{*} V \rightarrow \mathbb{C}$.

2. $V$ to be of quaternionic type if there exists a $G$-invariant skew-symmetric nondegenerate bilinear form $B: V \times \sigma_{G}^{*} V \rightarrow \mathbb{C}$.

3. $V$ to be of complex type if $V \nsubseteq \sigma_{G}^{*} \bar{V}$.

The abelian group generated by classes of irreducible complex representation of type $\mathbb{F}$ is denoted by $R(G, \mathbb{F})$.

Definition 2.20. If $V$ is a complex $G$-representation equipped with an anti-linear endomorphism $J_{V}$ such that $J_{V}(g v)=\sigma_{G}(g) J(v)$ and $J^{2}=-\mathrm{Id}_{V}$, then we say $V$ is a Quaternionic representation of $G$. Let $\mathcal{R e p}_{\mathbb{H}}(G)$ be the category of Quaternionic representations of $G$. A morphism between $V$ and $W \in \mathcal{R e p}_{\mathbb{H}}(G)$ is a linear transformation from $V$ to $W$ which commutes with $G$ and respect $J_{V}$ and $J_{W}$. We denote $\operatorname{Mor}(V, W)$ by $\operatorname{Hom}_{G}(V, W)^{\left(J_{V}, J_{W}\right)}$. An irreducible Quaternionic representation is an irreducible object in $\mathcal{R e p}_{\mathbb{H}}\left(G, \sigma_{G}\right)$. The Quaternionic representation group of $G$, denoted by $R H(G)$, is the Grothendieck group of $\mathcal{R e p}_{\mathbb{H}}(G)$. Let $R H(G, \mathbb{F})$ be the abelian group generated by the isomorphism classes of irreducible Quaternionic representations with $\mathbb{F}$ as the commuting field. 
Remark 2.21. The tensor product of two Quaternionic representations $V$ and $W$ is a Real representation as $J_{V} \otimes J_{W}$ is an anti-linear involution which is compatible with $\sigma_{G}$. Similarly the tensor product of a Real representation and a Quaternionic representation is a Quaternionic representation. To put it succinctly, $R R(G) \oplus R H(G)$ is a $\mathbb{Z}_{2}$-graded ring, with $R R(G)$ being the degree 0 piece and $R H(G)$ the degree -1 piece. Later on we will assign $R H(G)$ with a different degree so as to be compatible with the description of the coefficient ring $K R_{G}^{*}(\mathrm{pt})$.

Proposition 2.22. $R H(G)$, as an abelian group, is generated by the following

1. $\left[V \oplus \sigma_{G}^{*} \bar{V}\right]$ where $V$ is an irreducible complex representation of real type with $J(u, w)=$ $(-w, u)$. Its commuting field is $\mathbb{H}$.

2. $\left[V \oplus \sigma_{G}^{*} \bar{V}\right]$, where $V$ is an irreducible complex representation of complex type with $J(u, w)=$ $(-w, u)$. Its commuting field is $\mathbb{C}$.

3. $[V]$, where $V$ is an irreducible complex representation of quaternionic type. Its commuting field is $\mathbb{R}$.

Proof. The proof proceeds in the same fashion as does the proof for Proposition 2.16.

\section{Corollary 2.23.}

1. We have that $R R(G, \mathbb{R}) \cong R H(G, \mathbb{H}) \cong R(G, \mathbb{R}), R H(G, \mathbb{R}) \cong R R(G, \mathbb{H}) \cong R(G, \mathbb{H})$ and $R R(G, \mathbb{C}) \cong R H(G, \mathbb{C})$ as abelian groups.

2. $R R(G)$ is isomorphic to $R H(G)$ as abelian groups.

Proof. The result follows easily from Proposition 2.16, Definition 2.19 and Proposition 2.22.

\section{Proposition 2.24.}

1. The map $j: R H(G) \rightarrow R(G)$ which forgets the Quaternionic structure is injective.

2. Any complex G-representation which is a Quaternionic representation can only possess a unique Quaternionic structure up to isomorphisms of Quaternionic G-representations.

Proof. The proof proceeds in the same fashion as does the proof for Proposition 2.17. It suffices to show that, if $\eta: R(G) \rightarrow R H(G)$ is the map $[V] \mapsto\left[\left(V \oplus \sigma_{G}^{*} \bar{V}, \sigma_{V \oplus \iota_{G}^{*} \bar{V}}\right)\right]$, then $\eta \circ j$ amounts to multiplication by 2 , i.e. $\left(V \oplus \sigma_{G}^{*} \bar{V}, \sigma_{V \oplus \sigma_{G}^{*} \bar{V}} \cong(V \oplus V, J \oplus J)\right.$, where $\sigma_{V \oplus \sigma_{G}^{*} \bar{V}}(u, w)=(-w, u)$. This is true because of the Quaternionic $G$-representation isomorphism

$$
\begin{aligned}
f: & V \oplus \iota_{G}^{*} \bar{V} \rightarrow V \oplus V, \\
& (u, w) \mapsto(u+J w, i(u-J w)) .
\end{aligned}
$$

Example 2.25. We shall illustrate the similarities and differences of the various aforementioned representation groups with an example. Let $G=Q_{8} \times C_{3}$, the direct product of the quaternion group and the cyclic group of order 3, equipped with the trivial involution. There are 5 irreducible complex representations of $Q_{8}$, namely, the 4 1-dimensional representations which become trivial on restriction to the center $Z$ of $Q_{8}$ and descend to the 4 1-dimensional representations of $Q_{8} / Z \cong \mathbb{Z}_{2} \oplus \mathbb{Z}_{2}$, and the 2-dimensional faithful representation. We denote these representations by $1_{Q_{8}}, \rho_{(0,1)}, \rho_{(1,0)}, \rho_{(1,1)}$ and $\rho_{Q}$ respectively. Similarly, we let $1_{C_{3}}, \rho_{\zeta}$ and $\rho_{\zeta^{2}}$ be the three 1-dimensional complex irreducible representations of $C_{3}$. It can be easily seen that

$$
\begin{aligned}
& R\left(Q_{8}, \mathbb{R}\right)=\mathbb{Z} \cdot\left[1_{Q_{8}}\right] \oplus \mathbb{Z} \cdot\left[\rho_{(0,1)}\right] \oplus \mathbb{Z} \cdot\left[\rho_{(1,0)}\right] \oplus \mathbb{Z} \cdot\left[\rho_{(1,1)}\right], \\
& R\left(Q_{8}, \mathbb{C}\right)=0
\end{aligned}
$$




$$
\begin{aligned}
& R\left(Q_{8}, \mathbb{H}\right)=\mathbb{Z} \cdot\left[\rho_{Q}\right], \\
& R\left(C_{3}, \mathbb{R}\right)=\mathbb{Z} \cdot\left[1_{C_{3}}\right], \\
& R\left(C_{3}, \mathbb{C}\right)=\mathbb{Z} \cdot\left[\rho_{\zeta}\right] \oplus \mathbb{Z} \cdot\left[\rho_{\zeta^{2}}\right], \\
& R\left(C_{3}, \mathbb{H}\right)=0 .
\end{aligned}
$$

It follows that

$$
\begin{aligned}
& R(G, \mathbb{R})=\underset{x \in\left\{1_{Q_{8}}, \rho_{(0,1)}, \rho_{(1,0)}, \rho_{(1,1)}\right\}}{\bigoplus} \mathbb{Z} \cdot\left[x \widehat{\otimes} 1_{C_{3}}\right] \cong \mathbb{Z}^{4},
\end{aligned}
$$

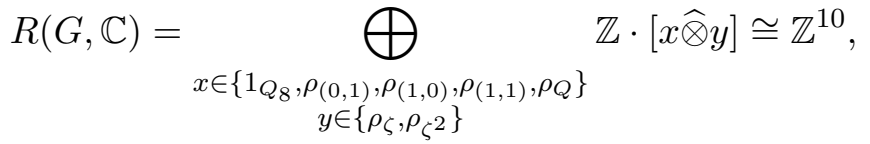

$$
\begin{aligned}
& R(G, \mathbb{H})=\mathbb{Z} \cdot\left[\rho_{Q} \widehat{\otimes} 1_{C_{3}}\right] \cong \mathbb{Z}, \\
& R R(G, \mathbb{R})=\underset{x \in\left\{1_{Q_{8}}, \rho_{(0,1)}, \rho_{(1,0)}, \rho_{(1,1)}\right\}}{\bigoplus} \mathbb{Z} \cdot\left[x \widehat{\otimes} 1_{C_{3}}\right] \cong \mathbb{Z}^{4},
\end{aligned}
$$

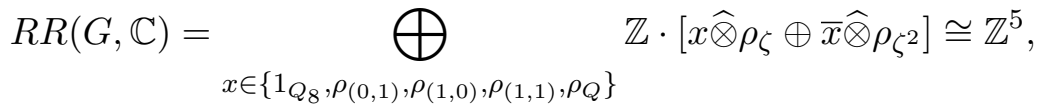

$$
\begin{aligned}
& R R(G, \mathbb{H})=\mathbb{Z} \cdot\left[\rho_{Q} \widehat{\otimes} 1_{C_{3}} \oplus \overline{\rho_{Q}} \widehat{\otimes} 1_{C_{3}}\right] \cong \mathbb{Z}, \\
& R H(G, \mathbb{R})=\mathbb{Z} \cdot\left[\rho_{Q} \widehat{\otimes} 1_{C_{3}}\right] \cong \mathbb{Z},
\end{aligned}
$$

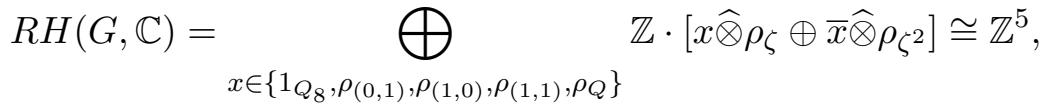

$$
\begin{aligned}
& R H(G, \mathbb{H})=\underset{x \in\left\{1_{\left.Q_{8}, \rho_{(0,1)}, \rho_{(1,0)}, \rho_{(1,1)}\right\}}\right.}{\bigoplus} \cdot\left[x \widehat{\otimes} 1_{C_{3}} \oplus \bar{x} \widehat{\otimes} 1_{C_{3}}\right] \cong \mathbb{Z}^{4}
\end{aligned}
$$

Some representations above should be equipped with suitable Real or Quaternionic structures given in Propositions 2.16 and 2.22. For example, the Real structure of $\rho_{Q} \widehat{\otimes} 1_{C_{3}} \oplus \overline{\rho_{Q}} \widehat{\otimes} 1_{C_{3}}$ in $R R(G, \mathbb{H})$ is given by swapping the two coordinates.

\section{$2.3 \quad K R$-theory}

$K R$-theory was first introduced by Atiyah in [2] and used to derive the 8-periodicity of $K O$ theory from the 2-periodicity of complex $K$-theory. $K R$-theory was motivated by the index theory of real elliptic operators.

\section{Definition 2.26.}

1. A Real space is a pair $\left(X, \sigma_{X}\right)$ where $X$ is a topological space equipped with an involutive homeomorphism $\sigma_{X}$, i.e. $\sigma_{X}^{2}=\operatorname{Id}_{X}$. We will sometimes suppress the notation $\sigma_{X}$ and simply use $X$ to denote the Real space, if there is no danger of confusion about the involutive homeomorphism. A Real pair is a pair $(X, Y)$ where $Y$ is a closed subspace of $X$ invariant under $\sigma_{X}$.

2. Let $\mathbb{R}^{p, q}$ be the Euclidean space $\mathbb{R}^{p+q}$ equipped with the involution which is identity on the first $q$ coordinates and negation on the last $p$-coordinates. Let $B^{p, q}$ and $S^{p, q}$ be the unit ball and sphere in $\mathbb{R}^{p, q}$ with the inherited involution.

3. A Real vector bundle (to be distinguished from the usual real vector bundle) over $X$ is a complex vector bundle $E$ over $X$ which itself is also a Real space with involutive homeomorphism $\sigma_{E}$ satisfying 
(a) $\sigma_{X} \circ p=p \circ \sigma_{E}$, where $p: E \rightarrow X$ is the projection map,

(b) $\sigma_{E} \operatorname{maps} E_{x}$ to $E_{\sigma_{X}(x)}$ anti-linearly.

A Quaternionic vector bundle (to be distinguished from the usual quaternionic vector bundle) over $X$ is a complex vector bundle $E$ over $X$ equipped with an anti-linear lift $\sigma_{E}$ of $\sigma_{X}$ such that $\sigma_{E}^{2}=-\operatorname{Id}_{E}$.

4. Let $X$ be a Real space. The ring $K R(X)$ is the Grothendieck group of the isomorphism classes of Real vector bundles over $X$, equipped with the usual product structure induced by tensor product of vector bundles over $\mathbb{C}$. The relative $K R$-theory for a Real pair $K R(X, Y)$ can be similarly defined. In general, the graded $K R$-theory ring of the Real pair $(X, Y)$ is given by

$$
K R^{*}(X, Y):=\bigoplus_{q=0}^{7} K R^{-q}(X, Y),
$$

where

$$
K R^{-q}(X, Y):=K R\left(X \times B^{0, q}, X \times S^{0, q} \cup Y \times B^{0, q}\right) .
$$

The ring structure of $K R^{*}$ is extended from that of $K R$, in a way analogous to the case of complex $K$-theory. The number of graded pieces, which is 8 , is a result of Bott periodicity for $K R$-theory (cf. [2]).

Note that when $\sigma_{X}=\operatorname{Id}_{X}$, then $K R(X) \cong K O(X)$. On the other hand, if $X \times \mathbb{Z}_{2}$ is given the involution which swaps the two copies of $X$, then $K R\left(X \times \mathbb{Z}_{2}\right) \cong K(X)$. Also, if $X$ is equipped with the trivial involution, then $K R\left(X \times S^{2,0}\right) \cong K S C(X)$, the Grothendieck group of homotopy classes of self-conjugate bundles over $X$ (cf. [2]). In this way, it is natural to view $K R$-theory as a unifying thread of $K O$-theory, $K$-theory and $K S C$-theory.

On top of the Real structure, we may further add compatible group actions and define equivariant $K R$-theory.

\section{Definition 2.27.}

1. A Real $G$-space $X$ is a quadruple $\left(X, G, \sigma_{X}, \sigma_{G}\right)$ where a group $G$ acts on $X$ and $\sigma_{G}$ is an involutive automorphism of $G$ such that

$$
\sigma_{X}(g \cdot x)=\sigma_{G}(g) \cdot \sigma_{X}(x)
$$

2. A Real $G$-vector bundle $E$ over a Real $G$-space $X$ is a Real vector bundle and a $G$-bundle over $X$, and it is also a Real $G$-space.

3. In a similar spirit, one can define equivariant $K R$-theory $K R_{G}^{*}(X, Y)$. Notice that the $G$ actions on $B^{0, q}$ and $S^{0, q}$ in the definition of $K R_{G}^{-q}(X, Y)$ are trivial.

\section{Definition 2.28.}

1. Let $K^{*}(+)$ be the complex $K$-theory of a point extended to a $\mathbb{Z}_{8}$-graded algebra over $K^{0}(\mathrm{pt}) \cong \mathbb{Z}$, i.e. $K^{*}(+) \cong \mathbb{Z}[\beta] / \beta^{4}-1$. Here $\beta \in K^{-2}(+)$ is the class of the reduced canonical bundle on $\mathbb{C P}^{1} \cong S^{2}$.

2. Let $\overline{\sigma_{X}^{*}}$ be the map defined on (equivariant) vector bundles on $X$ by $\overline{\sigma_{X}^{*}} E:=\sigma_{X}^{*} \bar{E}$. The involution induced by $\overline{\sigma_{X}^{*}}$ on $K_{G}^{*}(X)$ is also denoted by $\overline{\sigma_{X}^{*}}$ for simplicity. 
In the following proposition, we collect, for reader's convenience, some basic results of $K R$ theory (cf. [15, Section 2]), some of which are stated in the more general context of equivariant $K R$-theory.

\section{Proposition 2.29.}

1. We have

$$
K R^{*}(\mathrm{pt}) \cong \mathbb{Z}[\eta, \mu] /\left(2 \eta, \eta^{3}, \mu \eta, \mu^{2}-4\right),
$$

where $\eta \in K R^{-1}(\mathrm{pt}), \mu \in K R^{-4}(\mathrm{pt})$ represents the reduced Hopf bundles of $\mathbb{R} \mathbb{P}^{1}$ and $\mathbb{H} \mathbb{P}^{1}$ respectively.

2. Let $c: K R_{G}^{*}(X) \rightarrow K_{G}^{*}(X)$ be the homomorphism which forgets the Real structure of Real vector bundles, and $r: K_{G}^{*}(X) \rightarrow K R_{G}^{*}(X)$ be the realification map defined by $[E] \mapsto$ $\left[E \oplus \sigma_{G}^{*} \sigma_{X}^{*} \bar{E}\right]$. Then we have the following relations

(a) $c(1)=1, c(\eta)=0, c(\mu)=2 \beta^{2}$, where $\beta \in K^{-2}(\mathrm{pt})$ is the Bott class,

(b) $r(1)=2, r(\beta)=\eta^{2}, r\left(\beta^{2}\right)=\mu, r\left(\beta^{3}\right)=0$,

(c) $r(x c(y))=r(x) y, c r(x)=x+\sigma_{G}^{*} \overline{\sigma_{X}^{*}} x$ and $r c(y)=2 y$ for $x \in K_{G}^{*}(X)$ and $y \in$ $K R_{G}^{*}(X)$, where $K_{G}^{*}(X)$ is extended to a $\mathbb{Z}_{8}$-graded algebra by Bott periodicity.

Proof. (1) is given in [15, Section 2]. The proof of (2) is the same as in the nonequivariant case, which is given in [2].

Definition 2.30. A Quaternionic $G$-vector bundle over a Real space $X$ is a complex vector bundle $E$ equipped with an anti-linear vector bundle endomorphism $J$ on $E$ such that $J^{2}=-\operatorname{Id}_{E}$ and $J(g \cdot v)=\sigma_{G}(g) \cdot J(v)$. Let $K H_{G}^{*}(X)$ be the corresponding $K$-theory constructed using Quaternionic $G$-bundles over $X$.

By generalizing the discussion preceding Lemma 5.2 in [15] to the equivariant and graded setting, we define a natural transformation

$$
t: K H_{G}^{-q}(X) \rightarrow K R_{G}^{-q-4}(X)
$$

which sends

$$
0 \longrightarrow E_{1} \stackrel{f}{\longrightarrow} E_{2} \longrightarrow 0
$$

to

$$
0 \longrightarrow \pi^{*}\left(\mathbb{H} \otimes_{\mathbb{C}} E_{1}\right) \stackrel{g}{\longrightarrow} \pi^{*}\left(\mathbb{H} \otimes_{\mathbb{C}} E_{2}\right) \longrightarrow 0,
$$

where

1) $E_{i}, i=1,2$ are equivariant Quaternionic vector bundles on $X \times \mathbb{R}^{0, q}$ equipped with the Quaternionic structures $J_{E_{i}}$,

2) $f$ is an equivariant Quaternionic vector bundle homomorphism which is an isomorphism outside $X \times\{0\}$,

3) $\pi: X \times \mathbb{R}^{0, q+4} \rightarrow X \times \mathbb{R}^{0, q}$ is the projection map,

4) $\mathbb{H} \otimes_{\mathbb{C}} E_{i}$ is the equivariant Real vector bundles equipped with the Real structure $J \otimes J_{E_{i}}$,

5) $g$ is an equivariant Real vector bundle homomorphism defined by $g(v, w \otimes e)=(v, v w \otimes$ $f(e))$.

One can easily show by generalizing the discussion in the last section of [5] that

Proposition 2.31. $t$ is an isomorphism. 


\subsection{The module structure of $K R$-theory of compact simply-connected Lie groups}

The following structure theorem for $K R$-theory, due to Seymour, is crucial in his computation of $K R^{*}(\mathrm{pt})$-module structure of $K R^{*}(G)$.

Theorem 2.32 ([15, Theorem 4.2]). Suppose that $K^{*}(X)$ is a free abelian group and decomposed by the involution $\overline{\sigma_{X}^{*}}$ into the following summands

$$
K^{*}(X)=M_{+} \oplus M_{-} \oplus T \oplus \overline{\sigma_{X}^{*}} T,
$$

where $\overline{\sigma_{X}^{*}}$ is identity on $M_{+}$and negation on $M_{-}$. Suppose further that there exist $h_{1}, \ldots, h_{n} \in$ $K R^{*}(X)$ such that $c\left(h_{1}\right), \ldots, c\left(h_{n}\right)$ form a basis of the $K^{*}(+)$-module $K^{*}(+) \otimes\left(M_{+} \oplus M_{-}\right)$. Then, as $K R^{*}(\mathrm{pt})$-modules,

$$
K R^{*}(X) \cong F \oplus r\left(K^{*}(+) \otimes T\right),
$$

where $F$ is the free $K R^{*}(\mathrm{pt})$-module generated by $h_{1}, \ldots, h_{n}$.

Remark 2.33. If $T=0$, then the conditions in Theorem 2.32 are equivalent to $K^{*}(X)$ being free abelian and $c: K R^{*}(X) \rightarrow K^{*}(X)$ being surjective. In this special case the theorem implies that the map $K R^{*}(X) \otimes_{K R^{*}(\mathrm{pt})} K^{*}(\mathrm{pt}) \rightarrow K^{*}(X)$ defined by $a \otimes b \mapsto c(a) \cdot b$ is a ring isomorphism. This smacks of the definition of weakly equivariant formality (cf. Remark 2.8) and inspires us to define a similar notion for equivariant $K R$-theory (cf. Definition 4.2). We say a real space is real formal if it satisfies the conditions of Theorem 2.32.

Definition 2.34. Let $\sigma_{\mathbb{R}}$ be the complex conjugation of $U(n)$ or $U(\infty)$, and $\sigma_{\mathbb{H}}$ be the symplectic type involution $g \mapsto J_{m} \bar{g} J_{m}^{-1}$ on $U(2 m)$, or $U(2 \infty)$.

For any Real space $X, K R^{-1}(X)$ is isomorphic to the abelian group of equivariant homotopy classes of maps from $X$ to $U(\infty)$ which respect $\sigma_{X}$ and $\sigma_{\mathbb{R}}$ on $U(\infty)$. Similarly, $K R^{-5}(X)$, which is isomorphic to $K H^{-1}(X)$ by Proposition 2.31, is isomorphic to the abelian group of equivariant homotopy classes of maps from $X$ to $U(2 \infty)$ which respect $\sigma_{X}$ and $\sigma_{\mathbb{H}}$ on $U(2 \infty)$ (cf. remarks in the last two paragraphs of Appendix of [15]). We can define maps analogous to those in Definition 2.1 in the context of $K R$-theory.

Definition 2.35. Let $\delta_{\mathbb{R}}: R R(G) \rightarrow K R^{-1}(G)$ and $\delta_{\mathbb{H}}: R H(G) \rightarrow K R^{-5}(G)$ be group homomorphisms which send a Real (resp. Quaternionic) representation to the $K R$-theory element represented by its homotopy class.

Proposition 2.36. If $\rho \in R R(G)$, then $\delta_{\mathbb{R}}(\rho)$ is represented by the complex of vector bundles in Proposition 2.2 equipped with the Real structure given by

$$
\begin{gathered}
\iota: G \times \mathbb{R} \times V \rightarrow G \times \mathbb{R} \times V, \\
(g, t, v) \mapsto\left(\sigma_{G}(g), t, \bar{v}\right) .
\end{gathered}
$$

If $\rho \in R H(G)$, then $\delta_{\mathbb{H}}(\rho)$ can be similarly represented, with the Real structure replaced by the Quaternionic structure.

From this point on until the end of this section, we further assume that $G$ is connected and simply-connected unless otherwise specified. It is known that $R(G)$ is a polynomial ring over $\mathbb{Z}$ generated by fundamental representations, which are permuted by $\overline{\sigma_{G}^{*}}$ (cf. [15, Lemma 5.5]). Let

$$
R(G) \cong \mathbb{Z}\left[\varphi_{1}, \ldots, \varphi_{r}, \theta_{1}, \ldots, \theta_{s}, \gamma_{1}, \ldots, \gamma_{t}, \overline{\sigma_{G}^{*}} \gamma_{1}, \ldots, \overline{\sigma_{G}^{*}} \gamma_{t}\right]
$$

where $\varphi_{i} \in R R(G, \mathbb{R}), \theta_{j} \in R H(G, \mathbb{R}), \gamma_{k} \in R(G, \mathbb{C})$. Then $K^{*}(G)$, as a free abelian group, is generated by square-free monomials in $\delta\left(\varphi_{1}\right), \ldots, \delta\left(\varphi_{r}\right), \delta\left(\theta_{1}\right), \ldots, \delta\left(\theta_{s}\right), \delta\left(\gamma_{1}\right), \ldots, \delta\left(\gamma_{t}\right), \delta\left(\overline{\sigma_{G}^{*}} \gamma_{1}\right)$, $\ldots, \delta\left(\overline{\sigma_{G}^{*}} \gamma_{t}\right)$. Using Theorem 2.32, Seymour obtained 
Theorem 2.37 ([15, Theorem 5.6]).

1. Suppose that $\overline{\sigma_{G}^{*}}$ acts as identity on $R(G)$, i.e. any irreducible Real representation of $G$ is either of real type or quaternionic type. Then as $K R^{*}(\mathrm{pt})$-modules,

$$
K R^{*}(G) \cong \wedge_{K R^{*}(\mathrm{pt})}\left(\delta_{\mathbb{R}}\left(\varphi_{1}\right), \ldots, \delta_{\mathbb{R}}\left(\varphi_{r}\right), \delta_{\mathbb{H}}\left(\theta_{1}\right), \ldots, \delta_{\mathbb{H}}\left(\theta_{s}\right)\right) .
$$

2. More generally, $c\left(\delta_{\mathbb{R}}\left(\varphi_{i}\right)\right)=\delta\left(\varphi_{i}\right), c\left(\delta_{\mathbb{H}}\left(\theta_{j}\right)\right)=\beta^{2} \cdot \delta\left(\theta_{j}\right)$, and there exist $\lambda_{1}, \ldots, \lambda_{t} \in$ $K R^{0}(G)$ such that $c\left(\lambda_{k}\right)=\beta^{3} \cdot \delta\left(\gamma_{k}\right) \delta\left(\overline{\sigma_{G}^{*}} \gamma_{k}\right)$, and

$$
K R^{*}(G) \cong P \oplus T \cdot P
$$

as $K R^{*}(\mathrm{pt})$-module, where

- $P \cong \bigwedge_{K R^{*}(\mathrm{pt})}\left(\delta_{\mathbb{R}}\left(\varphi_{1}\right), \ldots, \delta_{\mathbb{R}}\left(\varphi_{r}\right), \delta_{\mathbb{H}}\left(\theta_{1}\right), \ldots, \delta_{\mathbb{H}}\left(\theta_{s}\right), \lambda_{1}, \ldots, \lambda_{t}\right)$,

- $T$ is the additive abelian group generated by the set

$$
\left\{r\left(\beta^{i} \cdot \delta\left(\gamma_{1}\right)^{\varepsilon_{1}} \cdots \delta\left(\gamma_{t}\right)^{\varepsilon_{t}} \delta\left(\overline{\sigma_{G}^{*}} \gamma_{1}\right)^{\nu_{1}} \cdots \delta\left(\overline{\sigma_{G}^{*}} \gamma_{t}\right)^{\nu_{t}}\right)\right\}
$$

where $\varepsilon_{1}, \ldots, \varepsilon_{t}, \nu_{1}, \ldots, \nu_{t}$ are either 0 or $1, \varepsilon_{k}$ and $\nu_{k}$ are not equal to 1 at the same time for $1 \leq k \leq t$, and the first index $k_{0}$ where $\varepsilon_{k_{0}}=1$ is less than the first index $k_{1}$ where $\nu_{k_{1}}=1$.

Moreover,

(a) $\lambda_{k}^{2}=0$ for all $1 \leq k \leq t$,

(b) $\delta_{\mathbb{R}}\left(\varphi_{i}\right)^{2}$ and $\delta_{\mathbb{H}}\left(\theta_{j}\right)^{2}$ are divisible by $\eta$.

Definition 2.38. Let $\omega_{t}:=\delta_{\epsilon_{t}, 1-\nu_{t}}$ and

$$
r_{i, \varepsilon_{1}, \ldots, \varepsilon_{t}, \nu_{1}, \ldots, \nu_{t}}:=r\left(\beta^{i} \cdot \delta\left(\gamma_{1}\right)^{\varepsilon_{1}} \cdots \delta\left(\gamma_{t}\right)^{\varepsilon_{t}} \delta\left(\overline{\sigma_{G}^{*}}\left(\gamma_{1}\right)\right)^{\nu_{1}} \cdots \delta\left(\overline{\sigma_{G}^{*}}\left(\gamma_{t}\right)\right)^{\nu_{t}}\right) \in T \text {. }
$$

\section{Corollary 2.39.}

1. $K R^{*}(G)$ is generated by $\delta_{\mathbb{R}}\left(\varphi_{1}\right), \ldots, \delta_{\mathbb{R}}\left(\varphi_{r}\right), \delta_{\mathbb{H}}\left(\theta_{1}\right), \ldots, \delta_{\mathbb{H}}\left(\theta_{s}\right), \lambda_{1}, \ldots, \lambda_{t}$ and $r_{i, \varepsilon_{1}, \ldots, \varepsilon_{t}, \nu_{1}, \ldots, \nu_{t}}$ $\in T$ as an algebra over $K R^{*}(\mathrm{pt})$.

2 .

$$
r_{i, \varepsilon_{1}, \ldots, \varepsilon_{t}, \nu_{1}, \ldots, \nu_{t}}^{2}= \begin{cases}\eta^{2} \lambda_{1}^{\omega_{1}} \cdots \lambda_{t}^{\omega_{t}}, & \text { if } r_{i, \varepsilon_{1}, \ldots, \varepsilon_{t}, \nu_{1}, \ldots, \nu_{t}} \text { is of degree }-1 \text { or }-5 \\ \pm \mu \lambda_{1}^{\omega_{1}} \cdots \lambda_{t}^{\omega_{t}}, & \text { if } r_{i, \varepsilon_{1}, \ldots, \varepsilon_{t}, \nu_{1}, \ldots, \nu_{t}} \text { is of degree }-2 \text { or }-6 \\ 0 & \text { otherwise }\end{cases}
$$

The sign depends on $i, \varepsilon_{1}, \ldots, \varepsilon_{t}, \nu_{1}, \ldots, \nu_{t}$ and can be determined using formulae from (2) of Proposition 2.29.

3. $r_{i, \varepsilon_{1}, \ldots, \varepsilon_{t}, \nu_{1}, \ldots, \nu_{t}} \eta=0$, and $r_{i, \varepsilon_{1}, \ldots, \varepsilon_{t}, \nu_{1}, \ldots, \nu_{t}} \mu=2 r_{i+2, \varepsilon_{1}, \ldots, \varepsilon_{t}, \nu_{1}, \ldots, \nu_{t}}$.

Proof. The Corollary follows easily from the various properties of the realification map and the complexification map in Proposition 2.29, and the fact that $c\left(\lambda_{k}\right)=\beta^{3} \cdot \delta\left(\gamma_{k}\right) \delta\left(\overline{\sigma_{G}^{*}} \gamma_{k}\right)$. For example,

$$
\begin{aligned}
r_{i, \varepsilon_{1}, \ldots, \varepsilon_{t}, \nu_{1}, \ldots, \nu_{t}} \eta & =r\left(\beta^{i} \cdot \delta\left(\gamma_{1}\right)^{\varepsilon_{1}} \cdots \delta\left(\gamma_{t}\right)^{\varepsilon_{t}} \delta\left(\overline{\sigma_{G}^{*}}\left(\gamma_{1}\right)\right)^{\nu_{1}} \cdots \delta\left(\overline{\sigma_{G}^{*}}\left(\gamma_{t}\right)\right)^{\nu_{t}} c(\eta)\right)=0, \\
r_{i, \varepsilon_{1}, \ldots, \varepsilon_{t}, \nu_{1}, \ldots, \nu_{t}} \mu & =r\left(\beta^{i} \cdot \delta\left(\gamma_{1}\right)^{\varepsilon_{1}} \cdots \delta\left(\gamma_{t}\right)^{\varepsilon_{t}} \delta\left(\overline{\sigma_{G}^{*}}\left(\gamma_{1}\right)\right)^{\nu_{1}} \cdots \delta\left(\overline{\sigma_{G}^{*}}\left(\gamma_{t}\right)\right)^{\nu_{t}} c(\mu)\right) \\
& =r_{i+2, \varepsilon_{1}, \ldots, \varepsilon_{t}, \nu_{1}, \ldots, \nu_{t} .}
\end{aligned}
$$


In fact Theorems 2.4 and 2.32 also yield the following description of module structure of $K R$-theory of a compact connected Real Lie group with torsion-free fundamental group with a restriction on the types of the Real representations.

Theorem 2.40. Let $G$ be a compact connected real Lie group with $\pi_{1}(G)$ torsion-free. Suppose that $R(G, \mathbb{C})=0$, i.e. $\overline{\sigma_{G}^{*}}$ acts as identity on $R(G)$. Then $K R^{*}(G)$ is isomorphic to $\wedge_{K R^{*}(\mathrm{pt})}^{*}\left(\operatorname{Im}\left(\widetilde{\delta}_{\mathbb{R}}\right), \operatorname{Im}\left(\widetilde{\delta}_{\mathbb{H}}\right)\right)$ as $K R^{*}(\mathrm{pt})$-modules.

As we see from Theorem 2.37 and Corollary 2.39, to get a full description of the ring structure of $K R^{*}(G)$, it remains to figure out $\delta_{\mathbb{R}}\left(\varphi_{i}\right)^{2}$ and $\delta_{\mathbb{H}}\left(\theta_{j}\right)^{2}$. We will, in the end, obtain formulae for the squares by way of computing the ring structure of $K R_{G}^{*}(G)$ and applying the forgetful map. In particular, we will show that $\delta_{\mathbb{R}}\left(\varphi_{i}\right)^{2}$ and $\delta_{\mathbb{H}}\left(\theta_{j}\right)^{2}$ in general are non-zero. So, unlike the complex $K$-theory, $K R^{*}(G)$ is not an exterior algebra in general. Nevertheless, $K R^{*}(G)$ is not far from being an exterior algebra, in the sense of the following

\section{Corollary 2.41.}

1. $K R^{*}(\mathrm{pt})_{2}$, which is the ring obtained by inverting the prime 2 in $K R^{*}(\mathrm{pt})$, is isomorphic to $\mathbb{Z}\left[\frac{1}{2}, \mu\right] /\left(\mu^{2}-4\right) \cong \mathbb{Z}\left[\frac{1}{2}, \beta^{2}\right] /\left(\left(\beta^{2}\right)^{2}-1\right)$.

2. Suppose that $R(G, \mathbb{C})=0 . K R^{*}(G)_{2}$, which is the ring obtained by inverting the prime 2 in $K R^{*}(G)$, is isomorphic to, as $K R^{*}(\mathrm{pt})_{2}$-algebra

$$
\bigwedge_{K R^{*}(\mathrm{pt})_{2}}\left(\delta_{\mathbb{R}}\left(\varphi_{1}\right), \ldots, \delta_{\mathbb{R}}\left(\varphi_{r}\right), \delta_{\mathbb{H}}\left(\theta_{1}\right), \ldots, \delta_{\mathbb{H}}\left(\theta_{s}\right)\right) .
$$

\section{$3 \quad$ The coefficient ring $K R_{G}^{*}(\mathrm{pt})$}

In this section, we assume that $G$ is a compact Real Lie group, and will prove a result on the coefficient ring $K R_{G}^{*}(\mathrm{pt})$. In [5], all graded pieces of $K R_{G}^{*}(\mathrm{pt})$ were worked out using Real Clifford $G$-modules. We record them in the following

Proposition 3.1. $K R_{G}^{-q}(\mathrm{pt})$, as abelian groups, for $0 \leq q \leq 7$, are isomorphic to $R R(G)$, $R R(G) / \rho(R(G)), R(G) / j(R H(G)), 0, R H(G), R H(G) / \eta(R(G)), R(G) / i(R R(G))$ and 0 respectively, where the maps $i, j, \rho, \eta$ are as in Propositions 2.17 and 2.24 .

Remark 3.2. Note from the above proposition that $K R_{G}^{0}(\mathrm{pt}) \oplus K R_{G}^{-4}(\mathrm{pt}) \cong R R(G) \oplus R H(G)$. In this way we can view $R R(G) \oplus R H(G)$ as a graded ring where $R R(G)$ is of degree 0 and $R H(G)$ of degree -4 .

\section{Proposition 3.3.}

1. Suppose $R(G, \mathbb{C})=0$. Then the map

$$
\begin{aligned}
f: \quad & (R R(G, \mathbb{R}) \oplus R H(G, \mathbb{R})) \otimes K R^{*}(\mathrm{pt}) \rightarrow K R_{G}^{*}(\mathrm{pt}), \\
& \rho_{1} \otimes x_{1} \oplus \rho_{2} \otimes x_{2} \mapsto \rho_{1} \cdot x_{1}+\rho_{2} \cdot x_{2}
\end{aligned}
$$

is an isomorphism of graded rings.

2. In general,

$$
\begin{aligned}
f: \quad & (R R(G, \mathbb{R}) \oplus R H(G, \mathbb{R})) \otimes K R^{*}(\mathrm{pt}) \oplus r\left(R(G, \mathbb{C}) \otimes K^{*}(+)\right) \rightarrow K R_{G}^{*}(\mathrm{pt}), \\
& \rho_{1} \otimes x_{1} \oplus \rho_{2} \otimes x_{2} \oplus r\left(\rho_{3} \otimes \beta^{i}\right) \mapsto \rho_{1} \cdot x_{1}+\rho_{2} \cdot x_{2}+r\left(\rho_{3} \cdot \beta^{i}\right)
\end{aligned}
$$

is an isomorphism of graded abelian groups. 
3. If $\rho$ is an irreducible complex representation of complex type, then $\eta r\left(\beta^{i} \cdot \rho\right)=0$ and $\mu r\left(\beta^{i} \cdot \rho\right)=2 r\left(\beta^{i+2} \cdot \rho\right)$.

Proof. The proposition follows by verifying the isomorphism in different degree pieces against the description in Proposition 3.1. For example, in degree 0,

$$
\begin{aligned}
& R R(G, \mathbb{R}) \otimes K R^{0}(\mathrm{pt}) \oplus R H(G, \mathbb{R}) \otimes K R^{-4}(\mathrm{pt}) \oplus r\left(R(G, \mathbb{C}) \otimes K^{0}(+)\right) \\
& \quad=R R(G, \mathbb{R}) \oplus R H(G, \mathbb{R}) \otimes \mathbb{Z} \mu \oplus R R(G, \mathbb{C}) \\
& \quad \cong R R(G, \mathbb{R}) \oplus R R(G, \mathbb{H}) \oplus R R(G, \mathbb{C}) \\
& \text { (if } \quad[V] \in R H(G, \mathbb{R}), \text { then }[V] \cdot \mu=[V \oplus V] \in R R(G, \mathbb{H})) \\
& \quad=R R(G)=K R_{G}^{0}(\mathrm{pt}) .
\end{aligned}
$$

(3) follows from Proposition 2.29.

Remark 3.4. In [5], $K R_{G}^{*}(X)$, where the $G$-action is trivial, is given as the following direct sum of abelian groups

$$
R R(G, \mathbb{R}) \otimes K R^{*}(X) \oplus R R(G, \mathbb{C}) \otimes K C^{*}(X) \oplus R R(G, \mathbb{H}) \otimes K H^{*}(X),
$$

where $K C^{*}(X)$ and $K H^{*}(X)$ are Grothendieck groups of the so-called 'Complex vector bundles' and 'Quaternionic vector bundles' of $X$. We find Proposition 3.3, which is motivated by this description, better because the ring structure of the coefficient ring is more apparent when cast in this light. The proposition is, as we will see in the next section, a consequence of a structure theorem of equivariant $K R$-theory (Theorem 4.5 ), and therefore still holds true if the point is replaced by any general space $X$ with trivial $G$-action.

\section{Equivariant $\boldsymbol{K} \boldsymbol{R}$-theory rings of compact simply-connected Lie groups}

Throughout this section we assume that $G$ is a compact, connected and simply-connected Real Lie group unless otherwise specified. We will prove the main result of this paper, Theorem 4.33, which gives the ring structure of $K R_{G}^{*}(G)$. Our strategy is outlined as follows.

1. We obtain a result on the structure of $K R_{G}^{*}(G)$ (Corollary 4.10) which is analogous to Theorem 2.37 and Proposition 3.3. We define $\delta_{\mathbb{R}}^{G}\left(\varphi_{i}\right), \delta_{\mathbb{H}}^{G}\left(\theta_{j}\right), \lambda_{k}^{G}$ and $r_{\rho, i, \varepsilon_{1}, \ldots, \varepsilon_{t}, \nu_{1}, \ldots, \nu_{t}}^{G}$ (cf. Definition 4.8 and Corollary 4.11), which generate $K R_{G}^{*}(G)$ as a $K R_{G}^{*}$ (pt)-algebra, as a result of Corollary 4.10. We show that $\left(\lambda_{k}^{G}\right)^{2}=0$ (cf. Proposition 4.13).

2. We compute the module structure of $K R_{\left(U(n), \sigma_{\mathbb{F}}\right)}^{*}\left(U(n), \sigma_{\mathbb{F}}\right)$ for $\mathbb{F}=\mathbb{R}$ and $\mathbb{H}$.

3. Let $T$ be the maximal torus of diagonal matrices in $U(n)$ and, by abuse of notation, $\sigma_{\mathbb{R}}$ be the inversion map on $T, \sigma_{\mathbb{H}}$ be the involution on $U(n) / T$ (where $n=2 m$ is even) defined by $g T \mapsto J_{m} \bar{g} T$. We show that the restriction map

$$
p_{G}^{*}: K R_{\left(U(n), \sigma_{\mathbb{R}}\right)}^{*}\left(U(n), \sigma_{\mathbb{R}}\right) \rightarrow K R_{\left(T, \sigma_{\mathbb{R}}\right)}^{*}\left(T, \sigma_{\mathbb{R}}\right)
$$

and the map

$$
q_{G}^{*}: K R_{\left(U(2 m), \sigma_{\mathbb{H}}\right)}^{*}\left(U(2 m), \sigma_{\mathbb{H}}\right) \rightarrow K R_{\left(U(2 m), \sigma_{\mathbb{H}}\right)}^{*}\left(U(2 m) / T \times T, \sigma_{\mathbb{H}} \times \sigma_{\mathbb{R}}\right)
$$

induced by the Weyl covering map $q_{G}: U(2 m) / T \times T \rightarrow U(2 m),(g T, t) \mapsto g t g^{-1}$, are injective. 
4. Let $\sigma_{n}$ be the class of the standard representation of $U(n)$. We pass the computation of the two squares $\delta_{\mathbb{R}}^{G}\left(\sigma_{n}\right)^{2} \in K R_{\left(U(n), \sigma_{\mathbb{R}}\right)}^{*}\left(U(n), \sigma_{\mathbb{R}}\right)$ and $\delta_{\mathbb{H}}^{G}\left(\sigma_{2 m}\right)^{2} \in K R_{\left(U(2 m), \sigma_{\mathbb{H}}\right)}^{*}\left(U(2 m), \sigma_{\mathbb{H}}\right)$ through the induced map $p_{G}^{*}$ and $q_{G}^{*}$ to their images and get equations (4.2) and (4.3) in Proposition 4.29.

5. Applying induced maps $\varphi_{i}^{*}$ and $\theta_{j}^{*}$ to equations (4.2) and (4.3) yields equations (4.4) and (4.5) in Theorem 4.30 which, together with Proposition 4.13 and some relations among $\eta, \mu$ and $r_{\rho, i, \varepsilon_{1}, \ldots, \varepsilon_{t}, \nu_{1}, \ldots, \nu_{t}}^{G}$ deduced from Proposition 2.29, describe completely the ring structure of $K R_{G}^{*}(G)$ (cf. Theorem 4.33).

\section{Remark 4.1.}

1. Seymour first suggested the analogues of Steps 3,4 and 5 in the ordinary $K R$-theory case in [15] in an attempt to compute $\delta_{\mathbb{R}}\left(\varphi_{i}\right)^{2}$ and $\delta_{\mathbb{H}}\left(\theta_{j}\right)^{2}$, but failed to establish Step 3, which he assumed to be true to make conjectures about $\delta_{\mathbb{R}}\left(\varphi_{i}\right)^{2}$.

2. In equivariant complex $K$-theory, $K_{G}^{*}(G / T \times T) \cong K_{T}^{*}(T)$ for any compact Lie group $G$, and the two maps $p_{G}^{*}$ (the restriction map induced by the inclusion $T \hookrightarrow G$ ) and $q_{G}^{*}$ which is induced by the Weyl covering map are the same. If $\pi_{1}(G)$ is torsion-free, then these two maps are shown to be injective (cf. [9]. In fact it is even shown there that the maps inject onto the Weyl invariants of $\left.K_{T}^{*}(T)\right)$. In the case of equivariant $K R$-theory, things are more complicated. First of all, while in the case where $\left(G, \sigma_{G}\right)=\left(U(n), \sigma_{\mathbb{R}}\right)$, it is true that $K R_{G}^{*}(G / T \times T) \cong K R_{T}^{*}(T)$, and $p_{G}^{*}$ and $q_{G}^{*}$ are the same, it is no longer true in the case where $\left(G, \sigma_{G}\right)=\left(U(2 m), \sigma_{\mathbb{H}}\right)$. In Step 3, we use $q_{G}^{*}$ for the quaternionic type involution case because we find that it admits an easier description than $p_{G}^{*}$ does. Second, we do not know whether $p_{G}^{*}$ and $q_{G}^{*}$ are injective for general compact Real Lie groups (equipped with any Lie group involution). For our purpose it is sufficient to show the injectivity results in Step 3.

\subsection{A structure theorem}

Definition 4.2. A $G$-space $X$ is a Real equivariantly formal space if

1) $G$ is a compact Real Lie group,

2) $X$ is a weakly equivariantly formal $G$-space, and

3) the forgetful map $K R_{G}^{*}(X) \rightarrow K R^{*}(X)$ admits a section $s_{R}: K R^{*}(X) \rightarrow K R_{G}^{*}(X)$ which is a $K R^{*}(\mathrm{pt})$-module homomorphism.

Remark 4.3. If $X$ is a weakly equivariantly formal $G$-space, then the forgetful map $K_{G}^{*}(X) \rightarrow$ $K^{*}(X)$ admits a (not necessarily unique) section $s: K^{*}(X) \rightarrow K_{G}^{*}(X)$ which is a group homomorphism.

Definition 4.4. For a section $s: K^{*}(X) \rightarrow K_{G}^{*}(X)$ (resp. $s_{R}: K R^{*}(X) \rightarrow K R_{G}^{*}(X)$ ) and $a \in K^{*}(X)$ (resp. $a \in K R^{*}(X)$ ), we call $s(a)$ (resp. $s_{R}(a)$ ) a (Real) equivariant lift of $a$, with respect to $s$ (resp. $s_{R}$ ).

We first prove a structure theorem of equivariant $K R$-theory of Real equivariantly formal spaces.

Theorem 4.5. Let $X$ be a Real equivariantly formal space. For any element $a \in K^{*}(X)$ (resp. $a \in K R^{*}(X)$ ), let $a_{G} \in K_{G}^{*}(X)$ (resp. $\left.a_{G} \in K R_{G}^{*}(X)\right)$ be a (Real) equivariant lift of a with respect to a group homomorphic section $s$ (resp. $s_{R}$ which is a $K R^{*}(\mathrm{pt})$-module homomorphism). Then the map

$$
\begin{aligned}
f: \quad & (R R(G, \mathbb{R}) \oplus R H(G, \mathbb{R})) \otimes K R^{*}(X) \oplus r\left(R(G, \mathbb{C}) \otimes K^{*}(X)\right) \rightarrow K R_{G}^{*}(X), \\
& \rho_{1} \otimes a_{1} \oplus r\left(\rho_{2} \otimes a_{2}\right) \mapsto \rho_{1} \cdot\left(a_{1}\right)_{G} \oplus r\left(\rho_{2} \cdot\left(a_{2}\right)_{G}\right) .
\end{aligned}
$$


is a group isomorphism. In particular, if $R(G, \mathbb{C})=0$, then $f$ is a $K R_{G}^{*}(\mathrm{pt})$-module isomorphism.

Proof. Consider the following $H(p, q)$-systems

$$
\begin{aligned}
& H R^{\alpha}(p, q):=K R^{-\alpha}\left(X \times S^{q, 0}, X \times S^{p, 0}\right) \cong K R^{-\alpha+p}\left(X \times S^{q-p, 0}\right), \\
& H^{\alpha}(p, q):=K^{-\alpha}\left(X \times S^{q, 0}, X \times S^{p, 0}\right) \cong K^{-\alpha+p}\left(X \times S^{q-p, 0}\right), \\
& H R_{G}^{\alpha}(p, q):=K R_{G}^{-\alpha}\left(X \times S^{q, 0}, X \times S^{p, 0}\right) \cong K R_{G}^{-\alpha+p}\left(X \times S^{q-p, 0}\right) .
\end{aligned}
$$

For the last $H(p, q)$-system, $G$ acts on $S^{q-p, 0}$ trivially. The spectral sequences induced by these $H(p, q)$-systems converge to $K R^{*}(X), K^{*}(X)$ and $K R_{G}^{*}(X)$ respectively (for the assertion for the first two $H(p, q)$-systems, see the proofs of Theorem 3.1 and Lemma 4.1 of [15]. That the third $H(p, q)$-system converges to $K R_{G}^{*}(X)$ follows from a straightforward generalization of the aforementioned proofs by adding equivariant structure throughout). Consider the two long exact sequences for the pair $\left(X \times B^{q-p, 0}, X \times S^{q-p, 0}\right)$, with the top exact sequence involving equivariant $K R$-theory and the bottom one ordinary $K R$-theory, and the vertical maps being forgetful maps. By applying the five-lemma, we have that each element in the first two $H(p, q)$ systems has a (Real) equivariant lift. Define a group homomorphism

$$
f(p, q):(R R(G, \mathbb{R}) \oplus R H(G, \mathbb{R})) \otimes H R^{\alpha}(p, q) \oplus r\left(R(G, \mathbb{C}) \otimes H^{\alpha}(p, q)\right) \rightarrow H R_{G}^{\alpha}(p, q)
$$

by

$$
\rho_{1} \otimes a_{1} \oplus r\left(\rho_{2} \otimes a_{2}\right) \mapsto \rho_{1} \cdot\left(a_{1}\right)_{G} \oplus r\left(\rho_{2} \cdot\left(a_{2}\right)_{G}\right) .
$$

As $R R(G, \mathbb{R}), R H(G, \mathbb{R})$ and $R(G, \mathbb{C})$ are free abelian groups, and tensoring free abelian groups and taking cohomology commute, $f$ is the abutment of $f(p, q)$. On the $E_{1}^{p, q}$-page, $f(p, q)$ becomes

$$
\begin{aligned}
f_{1}^{p, q}: & (R R(G, \mathbb{R}) \oplus R H(G, \mathbb{R})) \otimes K R^{-q}\left(X \times S^{1,0}\right) \oplus r\left(R(G, \mathbb{C}) \otimes K^{-q}\left(X \times S^{1,0}\right)\right) \\
& \rightarrow K R_{G}^{-q}\left(X \times S^{1,0}\right) .
\end{aligned}
$$

Note that $K^{-q}\left(X \times S^{1,0}\right) \cong K^{-q}(X) \oplus K^{-q}(X), K R^{-q}\left(X \times S^{1,0}\right) \cong K^{-q}(X)$, and $K R_{G}^{-q}(X \times$ $\left.S^{1,0}\right) \cong K_{G}^{-q}(X)$. With the above identification,

$$
\begin{aligned}
r: & R(G, \mathbb{C}) \otimes K^{-q}\left(X \times S^{1,0}\right) \rightarrow K R_{G}^{-q}\left(X \times S^{1,0}\right) \cong K_{G}^{-q}(X), \\
& \rho_{1} \otimes\left(a_{1}, 0\right) \oplus \rho_{2} \otimes\left(0, a_{2}\right) \mapsto \rho_{1} \cdot\left(a_{1}\right)_{G}+\overline{\sigma_{G}^{*}} \rho_{2} \cdot\left(\overline{\sigma_{G}^{*}} a_{2}\right)_{G} .
\end{aligned}
$$

So $r\left(R(G, \mathbb{C}) \otimes K^{-q}\left(X \times S^{1,0}\right)\right)=R(G, \mathbb{C}) \otimes K^{-q}(X) . \quad f_{1}^{p, q}$ is a group homomorphism from $(R R(G, \mathbb{R}) \oplus R H(G, \mathbb{R}) \oplus R(G, \mathbb{C})) \otimes K^{-q}(X) \cong R(G) \otimes K^{-q}(X)$ to $K_{G}^{-q}(X)$, which is an isomorphism by weak equivariant formality of $X$. It follows that $f$ is also an isomorphism. If $R(G, \mathbb{C})=0$, then by (1) of Proposition 3.3 and (3) of Definition $4.2, f$ is indeed a $K R_{G}^{*}(\mathrm{pt})$ module isomorphism.

Remark 4.6. The term 'Real equivariant formality' is suggested by the observation that, if $X$ is a Real equivariantly formal $G$-space and $R(G, \mathbb{C})=0$, then the map

$$
K R_{G}^{*}(X) \otimes_{R R(G, \mathbb{R}) \oplus R H(G, \mathbb{R})} \mathbb{Z} \rightarrow K R^{*}(X)
$$

induced by the forgetful map is a ring isomorphism, which smacks of the ring isomorphism in the definition of weak equivariant formality.

Lemma 4.7. $\delta_{\mathbb{R}}\left(\varphi_{i}\right), \delta_{\mathbb{H}}\left(\theta_{j}\right), \lambda_{k}$ and $r_{i, \varepsilon_{1}, \ldots, \varepsilon_{t}, \nu_{1}, \ldots, \nu_{t}} \in K R^{*}(G)$ all have Real equivariant lifts in $K R_{G}^{*}(G)$. Hence $G$ is a Real equivariantly formal space. 
Proof. A natural choice of a Real equivariant lift of $\delta_{\mathbb{R}}\left(\varphi_{i}\right)$ is represented by the complex of vector bundles in Proposition 2.2 equipped with both the Real structure and equivariant structure defined for $\delta_{\mathbb{R}}\left(\varphi_{i}\right)$ and $\delta_{G}(\varphi)$ respectively. These two structures are easily seen to be compatible. A Real equivariant lift of $\delta_{\mathbb{H}}\left(\theta_{j}\right)$ can be similarly defined. The class

$$
r\left(\beta^{i} \cdot \delta_{G}\left(\gamma_{1}\right)^{\varepsilon_{1}} \cdots \delta_{G}\left(\gamma_{t}\right)^{\varepsilon_{t}} \delta_{G}\left(\overline{\sigma_{G}^{*}} \gamma_{1}\right)^{\nu_{1}} \cdots \delta_{G}\left(\overline{\sigma_{G}^{*}} \gamma_{t}\right)^{\nu_{t}}\right)
$$

obviously is a Real equivariant lift of $r_{i, \varepsilon_{1}, \ldots, \varepsilon_{t}, \nu_{1}, \ldots, \nu_{t}}$. By adding the natural equivariant structure throughout the construction of $\lambda_{k}$ in the proof of Proposition 4.6 in [15], one can obtain a Real equivariant lift of $\lambda_{k}$.

Definition 4.8. We fix a choice of equivariant lift of any element $a \in K^{*}(G)$ by defining $\delta_{G}(\rho)$ to be the equivariant lift of $\delta(\rho)$. Similarly, we fix a choice of Real equivariant lift of $a \in K R^{*}(G)$ by defining $\delta_{\mathbb{R}}^{G}\left(\varphi_{i}\right), \delta_{\mathbb{H}}^{G}\left(\theta_{j}\right), \lambda_{k}^{G}$, and $r_{i, \varepsilon_{1}, \ldots, \varepsilon_{t}, \nu_{1}, \ldots, \nu_{t}}^{G}$ in the proof of Lemma 4.7 to be the Real equivariant lift of $\delta_{\mathbb{R}}\left(\varphi_{i}\right), \delta_{\mathbb{H}}\left(\theta_{j}\right), \lambda_{k}$ and $r_{i, \varepsilon_{1}, \ldots, \varepsilon_{t}, \nu_{1}, \ldots, \nu_{t}}$.

Remark 4.9. $\lambda_{k}^{G}$ satisfies $c\left(\lambda_{k}^{G}\right)=\beta^{3} \delta_{G}\left(\gamma_{k}\right) \delta_{G}\left(\overline{\sigma_{G}^{*}} \gamma_{k}\right)$.

Corollary 4.10. Let $G$ be a compact, connected and simply-connected Real Lie group. The map

$$
\begin{aligned}
f: & (R R(G, \mathbb{R}) \oplus R H(G, \mathbb{R})) \otimes K R^{*}(G) \oplus r\left(R(G, \mathbb{C}) \otimes K^{*}(G)\right) \rightarrow K R_{G}^{*}(G), \\
& \rho_{1} \otimes a_{1} \oplus r\left(\rho_{2} \otimes a_{2}\right) \mapsto \rho_{1} \cdot\left(a_{1}\right)_{G} \oplus r\left(\rho_{2} \cdot\left(a_{2}\right)_{G}\right)
\end{aligned}
$$

is a group isomorphism. Here $\left(a_{i}\right)_{G}$ is the (Real) equivariant lift defined as in Definition 4.8. In particular, if $R(G, \mathbb{C})=0$, then $f$ is an isomorphism of $K R_{G}^{*}(\mathrm{pt})$-modules from $K R_{G}^{*}(\mathrm{pt}) \otimes$ $K^{*}(G)$ to $K R_{G}^{*}(G)$.

Proof. The result follows from Theorem 4.5 and Lemma 4.7. In the special case where $R(G, \mathbb{C})=0, K R^{*}(G)$ is isomorphic to $K R^{*}(\mathrm{pt}) \otimes K^{*}(G)$ as $K R^{*}(\mathrm{pt})$-modules by (1) of Theorem 2.37, and applying Theorem 4.5 and Proposition 3.3 give $K R_{G}^{*}(G) \cong R R(G) \otimes K R^{*}(G) \cong$ $R R(G) \otimes K R^{*}(\mathrm{pt}) \otimes K^{*}(G) \cong K R_{G}^{*}(\mathrm{pt}) \otimes K^{*}(G)$. In this way $f$ is a $K R_{G}^{*}(\mathrm{pt})$-module isomorphism from $K R_{G}^{*}(\mathrm{pt}) \otimes K^{*}(G)$ to $K R_{G}^{*}(G)$.

Corollary 4.11. Let

$$
r_{\rho, i, \varepsilon_{1}, \ldots, \varepsilon_{t}, \nu_{1}, \ldots, \nu_{t}}^{G}:=r\left(\beta^{i} \cdot \rho \delta_{G}\left(\gamma_{1}\right)^{\varepsilon_{1}} \cdots \delta_{G}\left(\gamma_{t}\right)^{\varepsilon_{t}} \delta_{G}\left(\overline{\sigma_{G}^{*}} \gamma_{1}\right)^{\nu_{1}} \cdots \delta_{G}\left(\overline{\sigma_{G}^{*}} \gamma_{t}\right)^{\nu_{t}}\right),
$$

where $\rho \in R(G, \mathbb{C}) \oplus \mathbb{Z} \cdot \rho_{\text {triv }}$ and $\varepsilon_{1}, \ldots, \varepsilon_{t}, \nu_{1}, \ldots, \nu_{t}$ are as in Theorem 2.32. Then $K R_{G}^{*}(G)$, as an algebra over $K R_{G}^{*}(\mathrm{pt})$, is generated by $\delta_{\mathbb{R}}^{G}\left(\varphi_{1}\right), \ldots, \delta_{\mathbb{R}}^{G}\left(\varphi_{r}\right), \delta_{\mathbb{H}}^{G}\left(\theta_{1}\right), \ldots, \delta_{\mathbb{H}}^{G}\left(\theta_{s}\right), \lambda_{1}, \ldots, \lambda_{t}$, and $r_{\rho, i, \varepsilon_{1}, \ldots, \varepsilon_{t}, \nu_{1}, \ldots, \nu_{t}}^{G}$.

Remark 4.12. If $\rho=\rho_{\text {triv }}$, then $r_{\rho, i, \varepsilon_{1}, \ldots, \varepsilon_{t}, \nu_{1}, \ldots, \nu_{t}}^{G}=r_{i, \varepsilon_{1}, \ldots, \varepsilon_{t}, \nu_{1}, \ldots, \nu_{t}}^{G}$. If $\rho \in R(G, \mathbb{C})$, then $r_{\rho, i, \varepsilon_{1}, \ldots, \varepsilon_{t}, \nu_{1}, \ldots, \nu_{t}}^{G}$ comes from $r\left(R(G, \mathbb{C}) \otimes K^{*}(G)\right)$ in the decomposition of Theorem 4.5.

Now we are in a position to compute $\left(\lambda_{k}^{G}\right)^{2}$ by imitating the proof of Proposition 4.7 in [15].

Proposition 4.13. $\left(\lambda_{k}^{G}\right)^{2}=0$.

Proof. Consider the Real Lie group $\left(U(n) \times U(n), \sigma_{\mathbb{C}}\right)$, where $\sigma_{\mathbb{C}}\left(g_{1}, g_{2}\right)=\left(\overline{g_{2}}, \overline{g_{1}}\right)$. Let $p_{j}$ : $U(n) \times U(n) \rightarrow U(n)$ be the projection onto the $j$-th factor, and $u_{i}=p_{1}^{*}\left(\wedge^{i} \sigma_{n}\right), v_{i}=p_{2}^{*}\left(\wedge^{i} \sigma_{n}\right)$. Thus $\overline{\sigma_{\mathbb{C}}^{*}} u_{i}=v_{i}$. A decomposition of $K^{*}(U(n) \times U(n))$ by the induced involution $\overline{\sigma_{\mathbb{C}}^{*}}$ is given by $M \oplus T \oplus \overline{\sigma_{\mathbb{C}}^{*}} T$, where $M$ is the subalgebra generated by $\delta\left(u_{1}\right) \delta\left(v_{1}\right), \ldots, \delta\left(u_{n}\right) \delta\left(v_{n}\right)$. By Proposition 2.32, there exist $h_{1}, \ldots, h_{n} \in K R^{0}\left(U(n) \times U(n), \sigma_{\mathbb{C}}\right)$ such that $c\left(h_{i}\right)=\beta^{3} \delta\left(u_{i}\right) \delta\left(v_{i}\right)$, 
and $K R^{*}\left(U(n) \times U(n), \sigma_{\mathbb{C}}\right) \cong F \oplus r\left(K^{*}(+) \otimes T\right)$, where $F$ is the $K R^{*}(\mathrm{pt})$-module freely generated by monomials in $h_{1}, \ldots, h_{n}$. By Corollary 4.10 ,

$$
\begin{aligned}
& K R_{\left(U(n) \times U(n), \sigma_{\mathbb{C}}\right)}^{*}\left(U(n) \times U(n), \sigma_{\mathbb{C}}\right) \cong R R\left(U(n) \times U(n), \sigma_{\mathbb{C}}, \mathbb{R}\right) \otimes\left(F \oplus r\left(K^{*}(+) \otimes T\right)\right) \\
& \oplus r\left(R\left(U(n) \times U(n), \sigma_{\mathbb{C}}, \mathbb{C}\right) \otimes K^{*}(U(n) \times U(n))\right) .
\end{aligned}
$$

Let $h_{i}^{G}$ be the equivariant lift of $h_{i}$ as defined in Definition 4.8. So $c\left(h_{i}^{G}\right)=\beta^{3} \delta_{G}\left(u_{i}\right) \delta_{G}\left(v_{i}\right)$ and $c\left(\left(h_{i}^{G}\right)^{2}\right)=0$. Consequently $\left(h_{i}^{G}\right)^{2}=\eta k_{i}$ for some $k_{i} \in K R_{\left(U(n) \times U(n), \sigma_{\mathbb{C}}\right)}^{-7}\left(U(n) \times U(n), \sigma_{\mathbb{C}}\right)(\mathrm{cf}$. Gysin sequence (3.4) in [2] and its equivariant analogue). Since $\eta \cdot r(\cdot)=0$, we may assume that $k_{i}$ is from the component $R R\left(U(n) \times U(n), \sigma_{\mathbb{C}}, \mathbb{R}\right) \otimes F$. But the degree -7 piece of the later is 0 . So $\left(h_{i}^{G}\right)^{2}=0$.

Consider the map

$$
\begin{aligned}
\gamma_{k} \times \overline{\sigma_{\mathbb{C}}^{*}} \gamma_{k}: & \left(G, \sigma_{G}\right) \rightarrow\left(U(n) \times U(n), \sigma_{\mathbb{C}}\right), \\
& g \mapsto\left(\gamma_{k}(g), \overline{\gamma_{k}\left(\sigma_{G}(g)\right)}\right) .
\end{aligned}
$$

It can be easily seen that $\left(\gamma_{k} \times \overline{\sigma_{\mathbb{C}}^{*}} \gamma_{k}\right)^{*}\left(h_{1}^{G}\right)=\lambda_{k}^{G}$. So $\left(\lambda_{k}^{G}\right)^{2}=0$.

\subsection{The module structure of $K R_{\left(U(n), \sigma_{\mathbb{F}}\right)}^{*}\left(U(n), \sigma_{\mathbb{F}}\right)$}

Definition 4.14. Let $\sigma_{n}$ be (the class of) the standard representation of $U(n)$.

Proposition 4.15. $\sigma_{n}, \wedge^{2} \sigma_{n}, \ldots, \wedge^{n} \sigma_{n} \in R R\left(U(n), \sigma_{\mathbb{R}}, \mathbb{R}\right), \wedge^{2 i} \sigma_{2 m} \in R R\left(U(2 m), \sigma_{\mathbb{H}}, \mathbb{R}\right)$ and $\wedge^{2 i+1} \sigma_{2 m} \in R H\left(U(2 m), \sigma_{\mathbb{H}}, \mathbb{R}\right)$. Also, both $R\left(U(n), \sigma_{\mathbb{R}}, \mathbb{C}\right)$ and $R\left(U(2 m), \sigma_{\mathbb{H}}, \mathbb{C}\right)$ are 0 .

Proof. For the involution $\sigma_{\mathbb{R}}$ and $\wedge^{i} \sigma_{n}$, define the bilinear form

$$
\begin{aligned}
B_{\mathbb{R}}: & \wedge^{i} \sigma_{n} \times \sigma_{\mathbb{R}}^{*} \wedge^{i} \sigma_{n} \rightarrow \mathbb{C}, \\
& \left(v_{1} \wedge \cdots \wedge v_{i}, w_{1} \wedge \cdots \wedge w_{i}\right) \mapsto \operatorname{det}\left(\left\langle v_{j}, \overline{w_{k}}\right\rangle\right) .
\end{aligned}
$$

Obviously the form is $U(n)$-invariant, symmetric and non-degenerate. By Proposition 2.18, each of $\wedge^{i} \sigma_{n}, 1 \leq i \leq n$ is a Real representation of real type. Similarly, define, for the involution $\sigma_{\mathbb{H}}$ and $\wedge^{i} \sigma_{2 m}$, a bilinear form

$$
\begin{aligned}
B_{\mathbb{H}}: & \wedge^{i} \sigma_{2 m} \times \sigma_{\mathbb{H}}^{*} \wedge^{i} \sigma_{2 m} \rightarrow \mathbb{C}, \\
& \left(v_{1} \wedge \cdots \wedge v_{i}, w_{1} \wedge \cdots \wedge w_{i}\right) \mapsto \operatorname{det}\left(\left\langle J_{m} v_{j}, \overline{w_{k}}\right\rangle\right) .
\end{aligned}
$$

It is $U(n)$-invariant because

$$
\begin{aligned}
B_{\mathbb{H}}(g v, g w) & =\operatorname{det}\left(\left\langle J_{m} g v_{j}, \overline{J_{m} \bar{g} J_{m}^{-1} w_{k}}\right\rangle\right)=\operatorname{det}\left(\left\langle J_{m} g v_{j}, J_{m} g J_{m}^{-1} \overline{w_{k}}\right\rangle\right) \\
& =\operatorname{det}\left(\left\langle v_{j}, J_{m}^{-1} \overline{w_{k}}\right\rangle\right)=\operatorname{det}\left(\left\langle J_{m} v_{j}, \overline{w_{k}}\right\rangle\right) .
\end{aligned}
$$

Moreover

$$
\begin{aligned}
B_{\mathbb{H}}(v, w) & =\operatorname{det}\left(\left\langle J_{m} v_{j}, \overline{w_{k}}\right\rangle\right)=\operatorname{det}\left(\left\langle-v_{j}, J_{m} \overline{w_{k}}\right\rangle\right)=\operatorname{det}\left(-\overline{\left\langle J_{m} \overline{w_{k}}, v_{j}\right\rangle}\right) \\
& =\operatorname{det}\left(-\left\langle J_{m} w_{k}, \overline{v_{j}}\right\rangle\right)=(-1)^{i} B_{\mathbb{H}}(w, v) .
\end{aligned}
$$

So by Propositions 2.16, 2.18 and 2.22, $\wedge^{i} \sigma_{2 m}$ is a Real representation of real type when $i$ is even and a Quaternionic representation of real type when $i$ is odd. There are no complex representations of complex type because $\wedge^{i} \sigma_{n} \cong \sigma_{\mathbb{F}}^{*} \overline{\wedge^{i} \sigma_{n}}$ for $\mathbb{F}=\mathbb{R}$ and $\mathbb{H}$.

Lemma 4.16. $K R_{\left(U(n), \sigma_{\mathbb{F}}\right)}^{*}\left(U(n), \sigma_{\mathbb{F}}\right)$ is isomorphic to $\Omega_{K R_{\left(U(n), \sigma_{\mathbb{F}}\right)}^{*}(\mathrm{pt}) / K R^{*}(\mathrm{pt})}^{*}$ as $K R_{\left(U(n), \sigma_{\mathbb{F}}\right)}^{*}(\mathrm{pt})$ modules, where $\mathbb{F}=\mathbb{R}$ or $\mathbb{H}$. 
Proof. Theorem 4.5 implies that, $K R_{\left(U(n), \sigma_{\mathbb{R}}\right)}^{*}\left(U(n), \sigma_{\mathbb{R}}\right) \cong R R\left(U(n), \sigma_{\mathbb{R}}\right) \otimes K R^{*}\left(U(n), \sigma_{\mathbb{R}}\right)$ and $K R_{\left(U(n), \sigma_{\mathbb{H}}\right)}^{*}\left(U(n), \sigma_{\mathbb{H}}\right) \cong\left(R R\left(U(n), \sigma_{\mathbb{H}}, \mathbb{R}\right) \oplus R H\left(U(n), \sigma_{\mathbb{H}}, \mathbb{R}\right)\right) \otimes K R^{*}\left(U(n), \sigma_{\mathbb{H}}\right)$. Moreover, by Theorem 2.40 and Proposition 4.15,

$$
K R^{*}\left(U(n), \sigma_{\mathbb{R}}\right) \cong \bigwedge_{K R^{*}(\mathrm{pt})}\left(\delta_{\mathbb{R}}\left(\sigma_{n}\right), \ldots, \delta_{\mathbb{R}}\left(\wedge^{n} \sigma_{n}\right)\right)
$$

and

$$
K R^{*}\left(U(2 m), \sigma_{\mathbb{H}}\right) \cong \bigwedge_{K R^{*}(\mathrm{pt})}\left(\delta_{\mathbb{H}}\left(\sigma_{2 m}\right), \delta_{\mathbb{R}}\left(\wedge^{2} \sigma_{2 m}\right), \ldots, \delta_{\mathbb{R}}\left(\wedge^{2 m} \sigma_{2 m}\right)\right) .
$$

Putting all these together and applying Theorem 3.3, we get the desired conclusion.

Remark 4.17. As ungraded $K R^{*}(\mathrm{pt})$-modules, both

$$
K R_{\left(U(2 m), \sigma_{\mathbb{R}}\right)}^{*}\left(U(2 m), \sigma_{\mathbb{R}}\right) \quad \text { and } \quad K R_{\left(U(2 m), \sigma_{\mathbb{H}}\right)}^{*}\left(U(2 m), \sigma_{\mathbb{H}}\right)
$$

are isomorphic to $K_{U(2 m)}^{*}(U(2 m)) \otimes K R^{*}(\mathrm{pt})$.

\subsection{Injectivity results}

This step involves proving that the restriction map $p_{G}^{*}$ to the equivariant $K R$-theory of the maximal torus and the map $q_{G}^{*}$ induced by the Weyl covering map are injective.

Lemma 4.18. Let $G$ be a compact Lie group and $X$ a $G$-space. Let $i_{1}^{*}: K_{G}^{*}(X) \rightarrow K_{T}^{*}(X)$ be the map which restricts the $G$-action to T-action. Then

$$
i_{1}^{*} \otimes \operatorname{Id}_{R}: K_{G}^{*}(X) \otimes R \rightarrow K_{T}^{*}(X) \otimes R
$$

is injective for any ring $R$.

Proof. By [4, Proposition 4.9], $i_{1}^{*}$ is split injective. So is $i_{1}^{*} \otimes \operatorname{Id}_{R}$ for any ring $R$.

Lemma 4.19. Let $i_{2}^{*}: K_{T}^{*}(G) \rightarrow K_{T}^{*}(T) \cong R(T) \otimes K^{*}(T)$ be the map induced by the inclusion $T \hookrightarrow G$. Then

$$
i_{2}^{*} \delta_{T}(\rho)=\sum_{j=1}^{\operatorname{dim} \rho} e^{\tau_{j}} \otimes \delta\left(\tau_{j}\right) \in K_{T}^{-1}(T),
$$

where $\tau_{j}$ are the weights of $\rho$.

Proof. Let $V$ be the vector space underlying the representation $\rho$. $\delta_{T}(\rho)$ is represented by the complex of $T$-equivariant vector bundles

$$
\begin{aligned}
& 0 \longrightarrow G \times \mathbb{R} \times V \longrightarrow G \times \mathbb{R} \times V \longrightarrow 0 \\
& (g, t, v) \mapsto(g, t,-t \rho(g) v) \quad \text { if } t \geq 0, \\
& (g, t, v) \mapsto(g, t, t v) \quad \text { if } t \leq 0
\end{aligned}
$$

which, on restricting to

$$
0 \longrightarrow T \times \mathbb{R} \times V \longrightarrow T \times \mathbb{R} \times V \longrightarrow 0
$$

is decomposed into a direct sum of complexes of 1-dimensional $T$-equivariant vector bundles, each of which corresponds to a weight of $\rho$. 
Lemma 4.20. Let $G$ be a simply-connected, connected compact Lie group and $\rho_{1}, \ldots, \rho_{l}$ be its fundamental representations. Then

$$
i_{2}^{*}\left(\prod_{i=1}^{l} \delta_{T}\left(\rho_{i}\right)\right)=d_{G} \otimes \prod_{i=1}^{l} \delta\left(\varpi_{i}\right)
$$

where $\varpi_{i}$ the $i$-th fundamental weight and $d_{G}=\sum_{w \in W} \operatorname{sgn}(w) e^{w \cdot \sum_{i=1}^{l} \varpi_{i}} \in R(T)$ is the Weyl denominator.

Proof. Equation (4.1) follows from Lemma 4.19 and Lemma 3 of [1].

Lemma 4.21. Let $M$ be an $R$-module freely generated by $m_{1}, \ldots, m_{l}$, and $N$ an $R$-module. If $f: M \rightarrow N$ is an $R$-module homomorphism, and $r f\left(m_{1}\right) \wedge \cdots \wedge f\left(m_{l}\right) \in \bigwedge_{R}^{l} N$ is nonzero for all $r \in R \backslash\{0\}$, then

$$
\bigwedge^{*} f: \bigwedge_{R}^{*} M \rightarrow \bigwedge_{R}^{*} N
$$

is injective.

Proof. It suffices to show that $\bigwedge^{k} f$ is injective for $1 \leq k \leq l$. Suppose $I \subseteq\{1, \ldots, l\},|I|=k$, $m_{I}:=\bigwedge_{i \in I} m_{i}$ and $f\left(m_{I}\right):=\bigwedge_{i \in I} f\left(m_{i}\right)$. If $\sum_{|I|=k} r_{I} m_{I} \in \operatorname{ker}\left(\bigwedge^{k} f\right)$, then for any $J$ with $|J|=k$,

$$
0=\sum_{|I|=k} r_{I} f\left(m_{I}\right) \wedge f\left(m_{J^{c}}\right)=r_{J} f\left(m_{1}\right) \wedge \cdots \wedge f\left(m_{l}\right)
$$

Hence $\sum_{|I|=k} r_{I} m_{I}=0$ and the conclusion follows.

Lemma 4.22. Let $G$ be a simply-connected, connected and compact Lie group. Then the map

$$
i_{2}^{*} \otimes \operatorname{Id}_{R}: K_{T}^{*}(G) \otimes R \rightarrow K_{T}^{*}(T) \otimes R
$$

is injective for any ring $R$.

Proof. Note that

$$
\begin{aligned}
& K_{G}^{*}(G) \otimes_{R(G)} R(T) \rightarrow K_{T}^{*}(G), \\
& a \otimes \rho \mapsto i_{1}^{*}(a) \cdot \rho .
\end{aligned}
$$

is an $R(T)$-algebra isomorphism (cf. [12, Theorem 4.4]). Using Theorem 2.7, we have that $K_{T}^{*}(G)$ is isomorphic, as an $R(T)$-algebra, to $\bigwedge_{R(T)}^{*} M$, where $M$ is the $R(T)$-module freely generated by $\delta_{T}\left(\rho_{1}\right), \ldots, \delta_{T}\left(\rho_{l}\right)$. We also observe that $K_{T}^{*}(T)$ is isomorphic, as an $R(T)$-algebra, to $\bigwedge_{R(T)}^{*} N$, where $N$ is the $R(T)$-module freely generated by $\delta\left(\varpi_{1}\right), \ldots, \delta\left(\varpi_{l}\right)$. Note that the hypotheses of Lemma 4.21 are satisfied by $f=i_{2}^{*} \otimes \operatorname{Id}_{\mathbb{Z}_{m}}$ for any $m \geq 2$, as $r \prod_{i=1}^{l} i_{2}^{*} \delta_{T}\left(\rho_{i}\right)=r i_{2}^{*} \prod_{i=1}^{l} \delta_{T}\left(\rho_{i}\right)=$ $r d_{G} \otimes \prod_{i=1}^{l} \delta\left(\varpi_{i}\right)$ (by Lemma 4.20) is indeed nonzero for any nonzero $r$ in $\mathbb{Z}_{m}$ (the coefficients of $d_{G}$ are either 1 or -1 , so after reduction $\bmod m r d_{G}$ is still nonzero). Now that $i_{2}^{*} \otimes \mathbb{Z}_{m}$ is injective, so is $i_{2}^{*} \otimes \operatorname{Id}_{R}$ for any ring $R$. 


\section{Proposition 4.23.}

1. Let $G$ be a compact, connected and simply-connected Real Lie group such that $R R(G, \mathbb{C})=$ $R R(G, \mathbb{H})=0$ and there exists a maximal torus $T$ on which the involution acts by inversion. Then the restriction map $K R_{G}^{*}(G) \rightarrow K R_{T}^{*}(T)$ is injective.

2. The $\operatorname{map}_{G}^{*}: K R_{\left(U(n), \sigma_{\mathbb{R}}\right)}^{*}\left(U(n), \sigma_{\mathbb{R}}\right) \rightarrow K R_{\left(T, \sigma_{\mathbb{R}}\right)}^{*}\left(T, \sigma_{\mathbb{R}}\right)$ is injective.

Proof. By Corollary 4.10, $K R_{G}^{*}(G) \cong K_{G}^{*}(G) \otimes K R^{*}(\mathrm{pt})$ and $K R_{T}^{*}(T) \cong K_{T}^{*}(T) \otimes K R^{*}(\mathrm{pt})$ as $K R^{*}(\mathrm{pt})$-modules. Using this identification, we can as well identify the restriction map with $i^{*} \otimes \operatorname{Id}_{K R^{*}(\mathrm{pt})}$, where $i^{*}:=i_{2}^{*} \circ i_{1}^{*}$. Part (1) then follows from Lemmas 4.18 and 4.22. Part (2) is immediate once we apply Lemma 4.18 and note that the proofs of Lemmas 4.20 and 4.22 can be adapted to the case $G=U(n)$ by letting $\sigma_{n}, \ldots, \wedge^{n} \sigma_{n}$ play the role of the fundamental representations and their highest weights, the fundamental weights.

Lemma 4.24. $K R_{\left(U(2 m), \sigma_{\mathbb{H}}\right)}^{*}\left(U(2 m) / T, \sigma_{\mathbb{H}}\right) \cong \mathbb{Z}\left[e_{1}^{\mathbb{H}}, \ldots, e_{2 m}^{\mathbb{H}},\left(e_{1}^{\mathbb{H}} e_{2}^{\mathbb{H}} \cdots e_{2 m}^{\mathbb{H}}\right)^{-1}\right] \otimes K R^{*}(\mathrm{pt})$ as rings, where $e_{i}^{\mathbb{H}}$ lives in the degree -4 piece.

Proof. It is known that $K^{*}(U(2 m) / T) \cong \mathbb{Z}\left[\alpha_{1}, \ldots, \alpha_{2 m}\right] /\left(s_{i}-\left(\begin{array}{c}2 m \\ i\end{array}\right) \mid 1 \leq i \leq 2 m\right)$, where $\alpha_{i}=\left[U(2 m) \times_{T} \mathbb{C}_{e_{i}}\right]$ and $s_{i}$ is the $i$-th elementary symmetric polynomial (cf. [3, Proposition 2.7.13]). The induced map $\overline{\sigma_{\mathbb{H}}^{*}}$ acts as identity on $K^{*}(U(2 m) / T)$. The involution $\sigma_{\mathbb{H}}$ on the base lifts to a Quaternionic structure on the associated complex line bundle, so there exist $\alpha_{1}^{\mathbb{H}}, \ldots, \alpha_{2 m}^{\mathbb{H}} \in K R^{-4}(U(2 m) / T)$, such that their complexifications are $\beta^{2} \alpha_{1}, \ldots, \beta^{2} \alpha_{2 m} \in$ $K^{*}(U(2 m) / T)$. By Theorem $2.32, K R^{*}\left(U(2 m) / T, \sigma_{\mathbb{H}}\right)$ is a $K R^{*}(\mathrm{pt})$-module generated by polynomials in $\alpha_{1}^{\mathbb{H}}, \ldots, \alpha_{2 m}^{\mathbb{H}} \in K R^{-4}\left(U(2 m) / T, \sigma_{\mathbb{H}}\right)$. In fact it is not hard to see that $K R^{*}(U(2 m) / T$, $\left.\sigma_{\mathbb{H}}\right)$ is isomorphic to

$$
\mathbb{Z}\left[\alpha_{1}^{\mathbb{H}}, \ldots, \alpha_{2 m}^{\mathbb{H}}\right] \otimes K R^{*}(\mathrm{pt}) /\left(s_{2 k}-\left(\begin{array}{c}
2 m \\
2 k
\end{array}\right), s_{2 k-1}-\frac{1}{2} \mu\left(\begin{array}{c}
2 m \\
2 k-1
\end{array}\right) \mid 1 \leq k \leq m\right) .
$$

Also obvious is that each of $\alpha_{i}^{\mathbb{H}}$ has an equivariant lift $e_{i}^{\mathbb{H}} \in K R_{\left(U(2 m), \sigma_{\mathbb{H}}\right)}^{-4}\left(U(2 m) / T, \sigma_{\mathbb{H}}\right)$.

Now that all the hypotheses in Theorem 4.5 are satisfied, we can apply it, together with the fact that $R\left(U(2 m), \sigma_{\mathbb{H}}, \mathbb{C}\right)=0$ (cf. Proposition 4.15) to see that $K R_{\left(U(2 m), \sigma_{\mathbb{H}}\right)}^{*}\left(U(2 m) / T, \sigma_{\mathbb{H}}\right)$ is isomorphic to

$$
\left(R R\left(U(2 m), \sigma_{\mathbb{H}}, \mathbb{R}\right) \oplus R H\left(U(2 m), \sigma_{\mathbb{H}}, \mathbb{R}\right)\right) \otimes K R^{*}\left(U(2 m) / T, \sigma_{\mathbb{H}}\right)
$$

as $R R\left(U(2 m), \sigma_{\mathbb{H}}, \mathbb{R}\right) \oplus R H\left(U(2 m), \sigma_{\mathbb{H}}, \mathbb{R}\right)$-modules (actually as rings). Noting that

$$
R R\left(U(2 m), \sigma_{\mathbb{H}}, \mathbb{R}\right) \oplus R H\left(U(2 m), \sigma_{\mathbb{H}}, \mathbb{R}\right) \cong \mathbb{Z}\left[s_{1}, \ldots, s_{2 m}, s_{2 m}^{-1}\right]
$$

we establish the Lemma.

Proposition 4.25. $K R_{\left(U(2 m), \sigma_{\mathbb{H}}\right)}^{*}\left(U(2 m) / T \times T, \sigma_{\mathbb{H}} \times \sigma_{\mathbb{R}}\right)$ is isomorphic to

$$
\mathbb{Z}\left[e_{1}^{\mathbb{H}}, \ldots, e_{2 m}^{\mathbb{H}},\left(e_{1}^{\mathbb{H}} \cdots e_{2 m}^{\mathbb{H}}\right)^{-1}\right] \otimes K R^{*}\left(T, \sigma_{\mathbb{R}}\right)
$$

as graded rings.

Proof. First, by [14, Theorem 1], Proposition 3.3 and Lemma 4.24, $K R_{\left(U(2 m), \sigma_{\mathbb{H}}\right)}^{*}\left(U(2 m) / T, \sigma_{\mathbb{H}}\right)$ is a free $K R_{\left(U(2 m), \sigma_{\mathbb{H}}\right)}^{*}(\mathrm{pt})$-module. The same is also true of $K R_{\left(U(2 m), \sigma_{\mathbb{H}}\right)}^{*}\left(T, \sigma_{\mathbb{R}}\right)$ since, by Theorem 4.5, it is isomorphic to $R R\left(U(2 m), \sigma_{\mathbb{H}}\right) \otimes K R^{*}\left(T, \sigma_{\mathbb{R}}\right)$, which in turn is isomorphic to $K R_{\left(U(2 m), \sigma_{H}\right)}^{*}(\mathrm{pt}) \otimes K^{*}(T)$. The proposition follows from a version of Künneth formula for equivariant $K R$-theory. 
Remark 4.26. $K R_{\left(U(2 m), \sigma_{\mathbb{H}}\right)}^{*}\left(U(2 m) / T \times T, \sigma_{\mathbb{H}} \times \sigma_{\mathbb{R}}\right)$ is isomorphic to $K_{T}^{*}(T) \otimes K R^{*}(\mathrm{pt})$ and $K R_{\left(T, \sigma_{\mathbb{R}}\right)}^{*}\left(T, \sigma_{\mathbb{R}}\right)$ as ungraded $K R^{*}(\mathrm{pt})$-modules.

Proposition 4.27. The map $q_{G}^{*}$ is injective.

Proof. By Remarks 4.17 and $4.26, K R_{\left(U(2 m), \sigma_{\mathbb{H}}\right)}^{*}\left(U(2 m), \sigma_{\mathbb{H}}\right)$ and $K R_{\left(U(2 m), \sigma_{\mathbb{H}}\right)}^{*}(U(2 m) / T \times$ $\left.T, \sigma_{\mathbb{H}} \times \sigma_{\mathbb{R}}\right)$ are isomorphic to $K R_{\left(U(2 m), \sigma_{\mathbb{R}}\right)}^{*}\left(U(2 m), \sigma_{\mathbb{R}}\right)$ and $K R_{\left(T, \sigma_{\mathbb{R}}\right)}^{*}\left(T, \sigma_{\mathbb{R}}\right)$ respectively, as ungraded $K R^{*}(\mathrm{pt})$-modules. It is not hard to see that $q_{G}^{*}$ can be identified with $p_{G}^{*}$ under these isomorphisms. Now the result follows from Proposition 4.23.

\subsection{Squares of algebra generators of real and quaternionic types}

Lemma 4.28. $K R^{*}\left(T, \sigma_{\mathbb{R}}\right)$ is isomorphic to the exterior algebra over $K R^{*}(\mathrm{pt})$ generated by $\delta_{\mathbb{R}}\left(e_{1}\right), \ldots, \delta_{\mathbb{R}}\left(e_{n}\right)$, as $K R_{\left(T, \sigma_{\mathbb{R}}\right)}^{*}(\mathrm{pt})$-modules. Here $e_{i}$ is the 1-dimensional complex representation with weight being the $i$-th standard basis vector of the weight lattice. Moreover, $\delta_{\mathbb{R}}\left(e_{i}\right)^{2}=$ $\eta \delta_{\mathbb{R}}\left(e_{i}\right)$.

Proof. Since $R\left(T, \sigma_{\mathbb{R}}, \mathbb{C}\right)=0$, the module structure follows from Theorem 2.40. For the second part of the Lemma, see the appendix of [15].

Proposition 4.29. In $K R_{\left(U(n), \sigma_{\mathbb{R}}\right)}^{*}\left(U(n), \sigma_{\mathbb{R}}\right)$

$$
\delta_{\mathbb{R}}^{G}\left(\sigma_{n}\right)^{2}=\eta\left(\sigma_{n} \cdot \delta_{\mathbb{R}}^{G}\left(\sigma_{n}\right)-\delta_{\mathbb{R}}^{G}\left(\wedge^{2} \sigma_{n}\right)\right) .
$$

In $K R_{\left(U(2 m), \sigma_{\mathbb{H}}\right)}^{*}\left(U(2 m), \sigma_{\mathbb{H}}\right)$,

$$
\delta_{\mathbb{H}}^{G}\left(\sigma_{2 m}\right)^{2}=\eta\left(\sigma_{2 m} \cdot \delta_{\mathbb{H}}^{G}\left(\sigma_{2 m}\right)-\delta_{\mathbb{R}}^{G}\left(\wedge^{2} \sigma_{2 m}\right)\right) .
$$

Proof. Now that we have shown that $p_{G}^{*}$ and $q_{G}^{*}$ are injective by Propositions 4.23 and 4.27, we can compute $\delta_{\mathbb{R}}^{G}\left(\sigma_{n}\right)^{2}$ and $\delta_{\mathbb{H}}^{G}\left(\sigma_{2 m}\right)^{2}$ by passing the computation through $p_{G}^{*}$ and $q_{G}^{*}$ to their images. We prove the case $\mathbb{F}=\mathbb{R}$. The proof of the case $\mathbb{F}=\mathbb{H}$ is similar so we leave it to the reader. Note that

$$
\begin{aligned}
p_{G}^{*}\left(\delta_{\mathbb{R}}^{G}\left(\sigma_{n}\right)^{2}\right) & =p_{G}^{*}\left(\delta_{\mathbb{R}}^{G}\left(\sigma_{n}\right)\right)^{2}=\left(\sum_{i=1}^{n} e_{i} \otimes \delta_{\mathbb{R}}\left(e_{i}\right)\right)^{2} \\
& =\sum_{i=1}^{n} e_{i}^{2} \otimes \eta \delta_{\mathbb{R}}\left(e_{i}\right)+\sum_{i \neq j} e_{i} e_{j} \otimes \delta_{\mathbb{R}}\left(e_{i}\right) \delta_{\mathbb{R}}\left(e_{j}\right) \\
& \stackrel{\text { Lemma }}{=} \sum_{i=1}^{n} e_{i}^{2} \otimes \eta \delta_{\mathbb{R}}\left(e_{i}\right)+\sum_{i<j} e_{i} e_{j} \otimes\left(\delta_{\mathbb{R}}\left(e_{i}\right) \delta_{\mathbb{R}}\left(e_{j}\right)+\delta_{\mathbb{R}}\left(e_{j}\right) \delta_{\mathbb{R}}\left(e_{i}\right)\right) \\
& =\sum_{i=1}^{n} e_{i}^{2} \otimes \eta \delta_{\mathbb{R}}\left(e_{i}\right) .
\end{aligned}
$$

On the other hand,

$$
\begin{aligned}
p^{*}\left(\sigma_{n} \cdot \delta_{\mathbb{R}}^{G}\left(\sigma_{n}\right)\right) & =\left(\sum_{i=1}^{n} e_{i} \otimes 1\right)\left(\sum_{i=1}^{n} e_{i} \otimes \delta_{\mathbb{R}}\left(e_{i}\right)\right)=\sum_{1 \leq i, j \leq n} e_{i} e_{j} \otimes \delta_{\mathbb{R}}\left(e_{i}\right) \\
& =\sum_{i=1}^{n} e_{i}^{2} \otimes \delta_{\mathbb{R}}\left(e_{i}\right)+\sum_{i \neq j} e_{j} e_{i} \otimes \delta_{\mathbb{R}}\left(e_{i}\right)
\end{aligned}
$$




$$
\begin{aligned}
& =\sum_{i=1}^{n} e_{i}^{2} \otimes \delta_{\mathbb{R}}\left(e_{i}\right)+\sum_{1 \leq i<j \leq n} e_{j} e_{i} \otimes\left(\delta_{\mathbb{R}}\left(e_{i}\right)+\delta_{\mathbb{R}}\left(e_{j}\right)\right) \\
& =\sum_{i=1}^{n} e_{i}^{2} \otimes \delta_{\mathbb{R}}\left(e_{i}\right)+p_{G}^{*}\left(\delta_{\mathbb{R}}^{G}\left(\wedge^{2} \sigma_{n}\right)\right) .
\end{aligned}
$$

From the above equations we obtain

$$
p_{G}^{*}\left(\delta_{\mathbb{R}}^{G}\left(\sigma_{n}\right)^{2}\right)=\eta p_{G}^{*}\left(\sigma_{n} \cdot \delta_{\mathbb{R}}^{G}\left(\sigma_{n}\right)-\delta_{\mathbb{R}}^{G}\left(\wedge^{2} \sigma_{n}\right)\right) .
$$

By Proposition 4.23,

$$
\delta_{\mathbb{R}}^{G}\left(\sigma_{n}\right)^{2}=\eta\left(\sigma_{n} \cdot \delta_{\mathbb{R}}^{G}\left(\sigma_{n}\right)-\delta_{\mathbb{R}}^{G}\left(\wedge^{2} \sigma_{n}\right)\right) .
$$

Theorem 4.30. Let $G$ be a Real compact Lie group. Then

$$
\begin{aligned}
& \delta_{\mathbb{R}}^{G}\left(\varphi_{i}\right)^{2}=\eta\left(\varphi_{i} \cdot \delta_{\mathbb{R}}^{G}\left(\varphi_{i}\right)-\delta_{\mathbb{R}}^{G}\left(\wedge^{2} \varphi_{i}\right)\right), \\
& \delta_{\mathbb{H}}^{G}\left(\theta_{j}\right)^{2}=\eta\left(\theta_{j} \cdot \delta_{\mathbb{H}}^{G}\left(\theta_{j}\right)-\delta_{\mathbb{R}}^{G}\left(\wedge^{2} \theta_{j}\right)\right) .
\end{aligned}
$$

Proof. The induced map $\varphi_{i}^{*}: K R_{\left(U(n), \sigma_{\mathbb{R}}\right)}^{*}\left(U(n), \sigma_{\mathbb{R}}\right) \rightarrow K R_{\left(G, \sigma_{G}\right)}^{*}\left(G, \sigma_{G}\right)$ sends $\sigma_{n}$ to $\varphi_{i}$, and $\delta_{\mathbb{R}}^{G}\left(\sigma_{n}\right)$ to $\delta_{\mathbb{R}}^{G}\left(\varphi_{i}\right)$. Likewise, the induced map $\theta_{j}^{*}: K R_{\left(U(2 m), \sigma_{\mathbb{H}}\right)}^{*}\left(U(2 m), \sigma_{\mathbb{H}}\right)$ sends $\sigma_{2 m}$ to $\theta_{j}$, and $\delta_{\mathbb{H}}^{G}\left(\sigma_{2 m}\right)$ to $\delta_{\mathbb{H}}^{G}\left(\theta_{j}\right)$. The result now follows from Proposition 4.29.

To further express $\delta_{\mathbb{R}}^{G}\left(\wedge^{2} \varphi_{i}\right)$ and $\delta_{\mathbb{R}}^{G}\left(\wedge^{2} \theta_{j}\right)$ in terms of the module generators associated with the fundamental representations, we may use the following derivation property of $\delta_{\mathbb{R}}^{G}$ and $\delta_{\mathbb{H}}^{G}$.

Proposition 4.31. $\delta_{\mathbb{R}}^{G} \oplus \delta_{\mathbb{H}}^{G}$ is a derivation of the graded ring $R R(G) \oplus R H(G)$ (with $R R(G)$ of degree 0 and $R H(G)$ of degree -4$)$ taking values in the graded module $K R_{G}^{-1}(G) \oplus K R_{G}^{-5}(G)$.

Proof. We refer the reader to the proof of Proposition 3.1 of [9] with the definition of $\delta_{G}(\rho)$ given there (which is incorrect) replaced by the one in Definition 2.5. One just need to simply check that the homotopy $\rho_{s}$ in the proof for $t \geq 0$ intertwines with both $\sigma_{\mathbb{R}}$ and $\sigma_{\mathbb{H}}$.

Corollary 4.32. In $K R_{\left(U(n), \sigma_{\mathbb{R}}\right)}^{*}\left(U(n), \sigma_{\mathbb{R}}\right)$,

$$
\delta_{\mathbb{R}}^{G}\left(\wedge^{k} \sigma_{n}\right)^{2}=\eta \sum_{i=1}^{2 k} \wedge^{2 k-i} \sigma_{n} \cdot \delta_{\mathbb{R}}^{G}\left(\wedge^{i} \sigma_{n}\right) .
$$

In $K R_{\left(U(2 m), \sigma_{\mathbb{H}}\right)}^{*}\left(U(2 m), \sigma_{\mathbb{H}}\right)$,

$$
\begin{aligned}
& \delta_{\mathbb{H}}^{G}\left(\wedge^{2 k-1} \sigma_{2 m}\right)^{2}=\eta \sum_{j=1}^{2 k-1}\left(\wedge^{4 k-2 j-1} \sigma_{2 m} \cdot \delta_{\mathbb{H}}^{G}\left(\wedge^{2 j-1} \sigma_{2 m}\right)+\wedge^{4 k-2 j-2} \sigma_{2 m} \cdot \delta_{\mathbb{R}}^{G}\left(\wedge^{2 j} \sigma_{2 m}\right)\right), \\
& \delta_{\mathbb{R}}^{G}\left(\wedge^{2 k} \sigma_{2 m}\right)^{2}=\eta \sum_{j=1}^{2 k}\left(\wedge^{4 k-2 j+1} \sigma_{2 m} \cdot \delta_{\mathbb{H}}^{G}\left(\wedge^{2 j-1} \sigma_{2 m}\right)+\wedge^{4 k-2 j} \sigma_{2 m} \cdot \delta_{\mathbb{R}}^{G}\left(\wedge^{2 j} \sigma_{2 m}\right)\right) .
\end{aligned}
$$

Proof. By the definition,

$$
\left(\wedge^{k} \sigma_{n}\right)^{*}\left(\delta_{\mathbb{R}}^{G}\left(\wedge^{2} \sigma\left(\begin{array}{l}
n \\
k
\end{array}\right)\right)=\delta_{\mathbb{R}}^{G}\left(\wedge^{2}\left(\wedge^{k} \sigma_{n}\right)\right) .\right.
$$


By Exercise 15.32 of [10],

$$
\wedge^{2}\left(\wedge^{k} \sigma_{n}\right)=\bigoplus_{i} \Gamma_{\varpi_{k-2 i+1}+\varpi_{k+2 i-1}}
$$

By Giambelli's formula,

$$
\Gamma_{\varpi_{k-2 i+1}+\varpi_{k+2 i-1}}=\wedge^{k+2 i-1} \sigma_{n} \cdot \wedge^{k-2 i+1} \sigma_{n}-\wedge^{k+2 i} \sigma_{n} \cdot \wedge^{k-2 i} \sigma_{n} .
$$

By Proposition 4.31

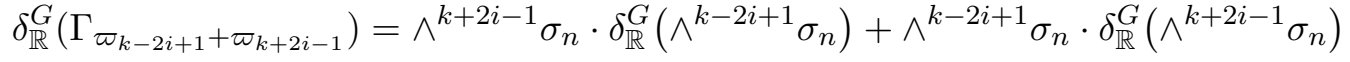

$$
\begin{aligned}
& -\wedge^{k+2 i} \sigma_{n} \cdot \delta_{\mathbb{R}}^{G}\left(\wedge^{k-2 i} \sigma_{n}\right)-\wedge^{k-2 i} \sigma_{n} \cdot \delta_{\mathbb{R}}^{G}\left(\wedge^{k+2 i} \sigma_{n}\right) .
\end{aligned}
$$

Now the first equation is immediate. The second and third equations can be derived similarly.

Putting together the previous results yields the following full description of the ring structure of $K R_{G}^{*}(G)$.

Theorem 4.33. Let $G$ be a simply-connected, connected and compact Real Lie group. Viewing $G$ as a Real G-space with adjoint action, we have

1. (Corollary 4.10) The map

$$
\begin{aligned}
f: & (R R(G, \mathbb{R}) \oplus R H(G, \mathbb{R})) \otimes K R^{*}(G) \oplus r\left(R(G, \mathbb{C}) \otimes K^{*}(G)\right) \rightarrow K R_{G}^{*}(G) \\
& \rho_{1} \otimes a_{1} \oplus r\left(\rho_{2} \otimes a_{2}\right) \mapsto \rho_{1} \cdot\left(a_{1}\right)_{G} \oplus r\left(\rho_{2} \cdot\left(a_{2}\right)_{G}\right)
\end{aligned}
$$

is a group isomorphism. In particular, if $R(G, \mathbb{C})=0$, then $f$ is an isomorphism of $K R_{G}^{*}(\mathrm{pt})$-modules.

2. (Corollary 4.11) $K R_{G}(G)$ is generated by $\delta_{\mathbb{R}}^{G}\left(\varphi_{1}\right), \ldots, \delta_{\mathbb{R}}^{G}\left(\varphi_{r}\right), \delta_{\mathbb{H}}^{G}\left(\theta_{1}\right), \ldots, \delta_{\mathbb{H}}^{G}\left(\theta_{s}\right), \lambda_{1}^{G}, \ldots, \lambda_{t}^{G}$ and $r_{\rho, i, \varepsilon_{1}, \ldots, \varepsilon_{t}, \nu_{1}, \ldots, \nu_{t}}^{G}$ as an algebra over $K R_{G}^{*}(\mathrm{pt})$. Moreover,

(a) (Proposition 4.13) $\left(\lambda_{k}^{G}\right)^{2}=0$ for all $1 \leq k \leq t$,

(b) $\quad\left(r_{\rho, i, \varepsilon_{1}, \ldots, \varepsilon_{t}, \nu_{1}, \ldots, \nu_{t}}^{G}\right)^{2}$

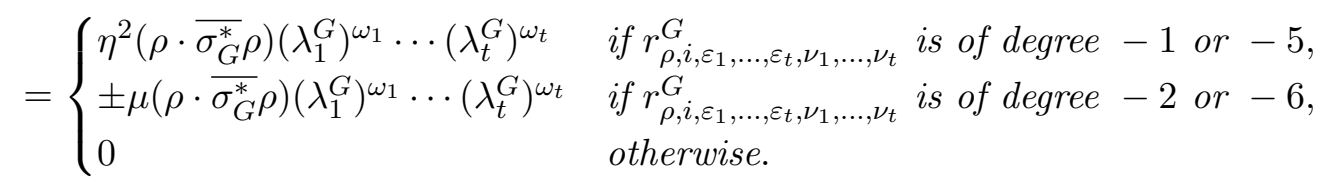

The sign can be determined using formulae in (2) of Proposition 2.29.

(c) $r_{\rho, i, \varepsilon_{1}, \ldots, \varepsilon_{t}, \nu_{1}, \ldots, \nu_{t}}^{G} \eta=0$, and $r_{\rho, i, \varepsilon_{1}, \ldots, \varepsilon_{t}, \nu_{1}, \ldots, \nu_{t}}^{G} \mu=2 r_{\rho, i+2, \varepsilon_{1}, \ldots, \varepsilon_{t}, \nu_{1}, \ldots, \nu_{t}}^{G}$,

(d) (Proposition 4.30) $\delta_{\mathbb{R}}^{G}\left(\varphi_{i}\right)^{2}=\eta\left(\varphi_{i} \cdot \delta_{\mathbb{R}}^{G}\left(\varphi_{i}\right)-\delta_{\mathbb{R}}^{G}\left(\wedge^{2} \varphi_{i}\right)\right)$,

$$
\delta_{\mathbb{H}}^{G}\left(\theta_{j}\right)^{2}=\eta\left(\theta_{j} \cdot \delta_{\mathbb{H}}^{G}\left(\theta_{j}\right)-\delta_{\mathbb{R}}^{G}\left(\wedge^{2} \theta_{j}\right)\right) .
$$

One can express $\delta_{\mathbb{R}}^{G}\left(\wedge^{2} \varphi_{i}\right)$ and $\delta_{\mathbb{H}}^{G}\left(\wedge^{2} \theta_{j}\right)$ in terms of the algebra generators using the derivation property of $\delta_{\mathbb{R}}^{G}$ and $\delta_{\mathbb{H}}^{G}$ (cf. Proposition 4.31 ).

Proof. Only (4.6) and (4.7) need explanation, but they are just equivariant analogues of Corollary 2.39 and follow from (2) of Proposition 2.29 and Remark 4.9. 


\section{Remark 4.34.}

1. $K R_{G}^{*}(\mathrm{pt})_{2}$, which is the ring obtained by inverting the prime 2 in $K R_{G}^{*}(\mathrm{pt})$, is isomorphic to

$$
\begin{gathered}
\left(R R\left(G, \sigma_{G}, \mathbb{R}\right) \oplus R H\left(G, \sigma_{G}, \mathbb{R}\right)\right) \otimes \mathbb{Z}\left[\frac{1}{2}, \beta^{2}\right] /\left(\left(\beta^{2}\right)^{2}-1\right) \\
\oplus r\left(R\left(G, \sigma_{G}, \mathbb{C}\right) \otimes \mathbb{Z}\left[\frac{1}{2}, \beta\right] /\left(\beta^{4}-1\right)\right) .
\end{gathered}
$$

2. If $R(G, \mathbb{C})=0$, then $K R_{G}^{*}(G)_{2}$, which is the ring obtained by inverting the prime 2 in $K R_{G}^{*}(G)$, is isomorphic to, as $K R_{G}^{*}(\mathrm{pt})_{2}$-algebra,

$$
\bigwedge_{K R_{G}^{*}(\mathrm{pt})_{2}}\left(\delta_{\mathbb{R}}^{G}\left(\varphi_{1}\right), \ldots, \delta_{\mathbb{R}}^{G}\left(\varphi_{r}\right), \delta_{\mathbb{H}}^{G}\left(\theta_{1}\right), \ldots, \delta_{\mathbb{H}}^{G}\left(\theta_{s}\right)\right) .
$$

\section{Applications and examples}

Applying the forgetful map $K R_{G}^{*}(G) \rightarrow K R^{*}(G)$ to Proposition 4.31 and Theorem 4.33, we solve the problem of finding a description of the ring structure of $K R^{*}(G)$ which was left open by Seymour in [15].

Theorem 5.1. Let $G$ be a simply-connected, connected and compact Real Lie group. Then

$$
\delta_{\mathbb{R}}\left(\varphi_{i}\right)^{2}=\eta\left(\operatorname{dim}\left(\varphi_{i}\right) \cdot \delta_{\mathbb{R}}\left(\varphi_{i}\right)-\delta_{\mathbb{R}}\left(\wedge^{2} \varphi_{i}\right)\right), \quad \delta_{\mathbb{H}}\left(\theta_{j}\right)^{2}=\eta \delta_{\mathbb{R}}\left(\wedge^{2} \theta_{j}\right) .
$$

One can express $\delta_{\mathbb{R}}\left(\wedge^{2} \varphi_{i}\right)$ and $\delta_{\mathbb{H}}\left(\wedge^{2} \theta_{j}\right)$ in terms of the generators in Proposition 2.37 using the derivation property of $\delta_{\mathbb{R}}$ and $\delta_{\mathbb{H}}$ got by applying the forgetful map to Proposition 4.31 . The above equations, together with Theorem 2.37 and Corollary 2.39, constitute a complete description of the ring structure of $K R^{*}(G)$.

Remark 5.2. Seymour's conjecture concerning $\delta_{\mathbb{R}}\left(\sigma_{n}\right)^{2}$ is true. However, his conjecture that if $x \in K R^{-5}(X)$, then $x^{2}=0$ is false, as evidenced by the ring structure of $K R^{*}\left(U(2 m), \sigma_{\mathbb{H}}\right)$.

Example 5.3. Let $G$ be a simply-connected, connected and compact Real Lie group with no fundamental representations of complex type. Equip $G$ with both the trivial $G$-action and the adjoint action. Both $K_{G_{\text {triv }}}^{*}(G)$ and $K_{G_{\text {Ad }}}^{*}(G)$ are isomorphic to $\Omega_{R(G) / \mathbb{Z}}^{*}$ as rings. On the other hand, though both $K R_{G_{\text {triv }}}^{*}(G)$ and $K R_{G_{\text {Ad }}}^{*}(G)$ are isomorphic to $\Omega_{K R_{G}^{*}(\mathrm{pt}) / K R^{*}(\mathrm{pt})}^{*}$ as $K R_{G}^{*}(\mathrm{pt})$ modules, they are not isomorphic as rings, as one can tell from the squares of the generators of both rings. For instance, in $K R_{G_{\text {triv }}}^{*}(G)$,

$$
\delta_{\mathbb{R}}^{G}\left(\varphi_{i}\right)^{2}=\eta\left(\operatorname{dim}\left(\varphi_{i}\right) \delta_{\mathbb{R}}^{G}\left(\varphi_{i}\right)-\delta_{\mathbb{R}}^{G}\left(\wedge^{2} \varphi_{i}\right)\right)
$$

whereas in $K R_{G_{\mathrm{Ad}}}^{*}(G)$,

$$
\delta_{\mathbb{R}}^{G}\left(\varphi_{i}\right)^{2}=\eta\left(\varphi_{i} \cdot \delta_{\mathbb{R}}^{G}\left(\varphi_{i}\right)-\delta_{\mathbb{R}}^{G}\left(\wedge^{2} \varphi_{i}\right)\right)
$$

In this example $K R$-theory can tell apart two different group actions, while $K$-theory cannot.

Example 5.4. Let $\left(G, \sigma_{G}\right)=(S p(2 m)$, Id $)$. Then $R(S p(2 m)) \cong \mathbb{Z}\left[\sigma_{2 m}^{1}, \sigma_{2 m}^{2}, \ldots, \sigma_{2 m}^{m}\right]$, where $\sigma_{2 m}^{i}$ is the class of the irreducible representation with highest weight $L_{1}+L_{2}+\cdots+L_{i}$. Note that $\sigma_{2 m}^{2 k-1} \in R H(S p(2 m), \mathrm{Id}, \mathbb{R}), \sigma_{2 m}^{2 k} \in R R(S p(2 m), \mathrm{Id}, \mathbb{R})$. Moreover, $\sigma_{2 m}^{i}+\wedge^{i-2} \sigma_{2 m}=\wedge^{i} \sigma_{2 m}$ for $1 \leq i \leq m$. The equivariant $K R$-theory $K R_{(S p(2 m), \text { Id })}^{*}(S p(2 m)$, Id $)$ is isomorphic to, as $K R_{(S p(2 m), \text { Id })}^{*}(\mathrm{pt})$-modules, the exterior algebra over $K R_{(S p(2 m), \text { Id })}^{*}(\mathrm{pt})$ generated by $\delta_{\mathbb{H}}^{G}\left(\sigma_{2 m}^{2 k-1}\right)$ and $\delta_{\mathbb{R}}^{G}\left(\sigma_{2 m}^{2 k}\right)$ for $1 \leq k \leq m$ by Theorem 4.33. The restriction map

$$
i^{*}: K R_{\left(U(2 m), \sigma_{\mathbb{H}}\right)}^{*}\left(U(2 m), \sigma_{\mathbb{H}}\right) \rightarrow K R_{(S p(2 m), \mathrm{Id})}^{*}(S p(2 m), \mathrm{Id})
$$


sends $\delta_{\mathbb{R}}^{G}\left(\wedge^{2 k} \sigma_{2 m}-\wedge^{2 k-2} \sigma_{2 m}\right)$ to $\delta_{\mathbb{R}}^{G}\left(\sigma_{2 m}^{2 k}\right)$ and $\delta_{\mathbb{H}}^{G}\left(\wedge^{2 k+1} \sigma_{2 m}-\wedge^{2 k-1} \sigma_{2 m}\right)$ to $\delta_{\mathbb{H}}^{G}\left(\sigma_{2 m}^{2 k+1}\right)$. Applying $i^{*}$ to the relevant equations in Corollary 4.32, we get

$$
\begin{aligned}
& \delta_{\mathbb{R}}^{G}\left(\sigma_{2 m}^{2 k}\right)^{2}=\eta \sum_{i=1}^{2 k}\left(\sigma_{2 m}^{4 k-2 i} \cdot \delta_{\mathbb{R}}^{G}\left(\sigma_{2 m}^{2 i}\right)+\sigma_{2 m}^{4 k-2 i+1} \cdot \delta_{\mathbb{H}}^{G}\left(\sigma_{2 m}^{2 i-1}\right)\right), \\
& \delta_{\mathbb{H}}^{G}\left(\sigma_{2 m}^{2 k-1}\right)^{2}=\eta \sum_{i=1}^{2 k-1}\left(\sigma_{2 m}^{4 k-2-2 i} \cdot \delta_{\mathbb{R}}^{G}\left(\sigma_{2 m}^{2 i}\right)+\sigma_{2 m}^{4 k-1-2 i} \cdot \delta_{\mathbb{H}}^{G}\left(\sigma_{2 m}^{2 i-1}\right)\right) .
\end{aligned}
$$

Example 5.5. Let $\left(G, \sigma_{G}\right)=\left(G_{2}, \mathrm{Id}\right)$. Then $R R\left(G_{2}\right) \cong \mathbb{Z}\left[\sigma_{1}, \sigma_{2}\right]$, where $\sigma_{1}$ and $\sigma_{2}$ are the classes of irreducible representations of dimensions 7 and 14, respectively. Note that both $\sigma_{1}$ and $\sigma_{2}$ are in $R R\left(G_{2}, \mathrm{Id}, \mathbb{R}\right)$, and that $\wedge^{2} \sigma_{1}=\sigma_{1}+\sigma_{2}, \wedge^{2} \sigma_{2}=\sigma_{1}^{3}-\sigma_{1}^{2}-2 \sigma_{1} \sigma_{2}-\sigma_{1}$. The equivariant $K R$-theory $K R_{\left(G_{2}, \mathrm{Id}\right)}^{*}\left(G_{2}, \mathrm{Id}\right)$ is isomorphic to, as $K R_{\left(G_{2}, \mathrm{Id}\right)}^{*}(\mathrm{pt})$-modules, the exterior algebra over $K R_{\left(G_{2}, \mathrm{Id}\right)}^{*}(\mathrm{pt})$ generated by $\delta_{\mathbb{R}}^{G}\left(\sigma_{1}\right)$ and $\delta_{\mathbb{R}}^{G}\left(\sigma_{2}\right)$, by Theorem 4.5. Using Theorem 4.30 and Proposition 4.31, we have

$$
\delta_{\mathbb{R}}^{G}\left(\sigma_{1}\right)^{2}=\eta\left(\left(\sigma_{1}-1\right) \cdot \delta_{\mathbb{R}}^{G}\left(\sigma_{1}\right)+\delta_{\mathbb{R}}^{G}\left(\sigma_{2}\right)\right), \quad \delta_{\mathbb{R}}^{G}\left(\sigma_{2}\right)^{2}=\eta\left(\left(\sigma_{1}^{2}-1\right) \cdot \delta_{\mathbb{R}}^{G}\left(\sigma_{1}\right)+\sigma_{2} \cdot \delta_{\mathbb{R}}^{G}\left(\sigma_{2}\right)\right) .
$$

\section{Acknowledgements}

The author would like to thank Professor Reyer Sjamaar for suggesting this problem, painstakingly proofreading the manuscript, his patient guidance and encouragement throughout the course of this project. He also thanks the referees for their critical comments, pointing out the relevance of the work [7] and a mistake in the definition of $\varphi(d \rho)$ in [9] to him.

\section{References}

[1] Atiyah M.F., On the $K$-theory of compact Lie groups, Topology 4 (1965), 95-99.

[2] Atiyah M.F., K-theory and reality, Quart. J. Math. Oxford Ser. (2) 17 (1966), 367-386.

[3] Atiyah M.F., K-theory, W.A. Benjamin, Inc., New York - Amsterdam, 1967.

[4] Atiyah M.F., Bott periodicity and the index of elliptic operators, Quart. J. Math. Oxford Ser. (2) 19 (1968), $113-140$.

[5] Atiyah M.F., Segal G.B., Equivariant K-theory and completion, J. Differential Geometry 3 (1969), 1-18.

[6] Bourbaki N., Lie groups and Lie algebras, Chapters 7-9, Elements of Mathematics (Berlin), Springer-Verlag, Berlin, 2005.

[7] Bousfield A.K., On the 2-adic K-localizations of H-spaces, Homology, Homotopy Appl. 9 (2007), 331-366.

[8] Bröcker T., tom Dieck T., Representations of compact Lie groups, Graduate Texts in Mathematics, Vol. 98, Springer-Verlag, New York, 1985.

[9] Brylinski J.L., Zhang B., Equivariant K-theory of compact connected Lie groups, K-Theory 20 (2000), 23-36, dg-ga/9710035.

[10] Fulton W., Harris J., Representation theory, Graduate Texts in Mathematics, Vol. 129, Springer-Verlag, New York, 1991.

[11] Harada M., Landweber G.D., Surjectivity for Hamiltonian G-spaces in K-theory, Trans. Amer. Math. Soc. 359 (2007), 6001-6025, math.SG/0503609.

[12] Harada M., Landweber G.D., Sjamaar R., Divided differences and the Weyl character formula in equivariant K-theory, Math. Res. Lett. 17 (2010), 507-527, arXiv:0906.1629.

[13] Hodgkin L., On the $K$-theory of Lie groups, Topology 6 (1967), 1-36.

[14] Pittie H.V., Homogeneous vector bundles on homogeneous spaces, Topology 11 (1972), 199-203.

[15] Seymour R.M., The real K-theory of Lie groups and homogeneous spaces, Quart. J. Math. Oxford Ser. (2) 24 (1973), 7-30. 